\title{
Donor and host B7-H4 expression negatively regulates acute graft-versus- host disease lethality
}

Asim Saha, ${ }^{1}$ Patricia A. Taylor, ${ }^{1}$ Christopher J. Lees, ${ }^{1}$ Angela Panoskaltsis-Mortari, ${ }^{1}$ Mark J. Osborn, Colby J. Feser, ${ }^{1}$ Govindarajan Thangavelu, ${ }^{1}$ Wolfgang Melchinger, ${ }^{2}$ Yosef Refaeli, ${ }^{3}$ Geoffrey R. Hill, ${ }^{4}$ David H. Munn, ${ }^{5}$ William J. Murphy, ${ }^{6}$ Jonathan S. Serody, ${ }^{7}$ Ivan Maillard, ${ }^{8}$ Katharina Kreymborg, ${ }^{9}$ Marcel van den Brink, ${ }^{9}$ Chen Dong, ${ }^{10}$ Shuyu Huang, ${ }^{11}$ Xingxing Zang, ${ }^{11}$ James P. Allison, ${ }^{12}$ Robert Zeiser, ${ }^{2}$ and Bruce R. Blazar ${ }^{1}$

'Masonic Cancer Center and Department of Pediatrics, Division of Blood and Marrow Transplantation, University of Minnesota, Minneapolis, Minnesota, USA. '2Department of Hematology, Oncology, and Stem-Cell Transplantation, Freiburg University Medical Center, Albert Ludwigs University Freiburg, Freiburg, Germany. ${ }^{3}$ Department of Dermatology, University of Colorado, Aurora, Colorado, USA. ${ }^{4}$ Fred Hutchinson Cancer Research Center, University of Washington, Seattle, Washington, USA. ${ }^{5}$ Department of Pediatrics, Georgia Health Sciences University, Augusta, Georgia, USA. ${ }^{6}$ Department of Dermatology, UC Davis School of Medicine, Sacramento, California, USA. ${ }^{7}$ Department of Medicine, University of North Carolina, Chapel Hill, North Carolina, USA. ${ }^{8}$ Department of Internal Medicine, University of Michigan, Ann Arbor, Michigan, USA. ${ }^{9}$ Department of Immunology and Medicine, Memorial Sloan Kettering Cancer Center, New York, New York, USA. ${ }^{10}$ Institute for Immunology and School of Medicine, Tsinghua University, Beijing, China. ${ }^{11}$ Department of Microbiology and Immunology, Albert Einstein College of Medicine, Bronx, New York, USA. ${ }^{12}$ Department of Immunology, The University of Texas MD Anderson Cancer Center, Houston, Texas, USA.

B7-H4 is a negative regulatory B7 family member. We investigated the role of host and donor B7-H4 in regulating acute graft-versus-host disease (GVHD). Allogeneic donor T cells infused into B7$\mathrm{H}^{-/-}$versus WT recipients markedly accelerated CVHD-induced lethality. Chimera studies pointed toward B7-H4 expression on host hematopoietic cells as more critical than parenchymal cells in controlling GVHD. Rapid mortality in B7- $\mathrm{H4}^{-/-}$recipients was associated with increased donor T cell expansion, gut $\mathrm{T}$ cell homing and loss of intestinal epithelial integrity, increased $\mathrm{T}$ effector function (proliferation, proinflammatory cytokines, cytolytic molecules), and reduced apoptosis. Higher metabolic demands of rapidly proliferating donor T cells in B7- $4^{-/-}$versus WT recipients required multiple metabolic pathways, increased extracellular acidification rates (ECARs) and oxygen consumption rates (OCRs), and increased expression of fuel substrate transporters. During CVHD, B7-H4 expression was upregulated on allogeneic WT donor T cells. B7- $4^{-1-}$ donor T cells given to WT recipients increased CVHD mortality and had function and biological properties similar to WT $\mathrm{T}$ cells from allogeneic $\mathrm{B} 7-\mathrm{H}^{-/-}$recipients. Graft-versus-leukemia responses were intact regardless as to whether $\mathrm{B} 7-\mathrm{H}^{-/-}$mice were used as hosts or donors. Taken together, these data provide new insights into the negative regulatory processes that control CVHD and provide support for developing therapeutic strategies directed toward the B7-H4 pathway.

Conflict of interest: The authors have declared that no conflict of interest exists.

Copyright: (c) 2019, American Society for Clinical Investigation.

Submitted: January 26, 2019

Accepted: August 23, 2019

Published: October 3, 2019

Reference information: JCl Insight. 2019;4(19):e127716.

https://doi.org/10.1172/jci.

insight.127716.

\section{Introduction}

Allogeneic hematopoietic stem cell transplantation (HSCT) is the preferred treatment for many hematological malignancies. However, graft-versus-host disease (GVHD), induced by donor $\mathrm{T}$ cells that recognize host alloantigenic disparities, is a frequent cause of morbidity and mortality following allogeneic HSCT $(1,2)$. The balance between costimulatory and coinhibitory pathways determines the fate of $\mathrm{T}$ cells following $\mathrm{T}$ cell receptor ligation. During GVHD, coinhibitory pathways can be upregulated, reducing injury to the host during acute GVHD (1).

The B7 family members play a critical role in both positive and negative regulation of immune responses by engaging a variety of receptors on lymphocytes. B7-H4 (B7S1, B7x, VTCN1) is a type-1 
transmembrane protein and a highly conserved B7 family member between mice and humans (3-5). B7-H4 encodes a glycosylated protein and plays an inhibitory role in T-cell function $(4,5)$. Expression of B7-H4 mRNA has been detected at low levels in a variety of tissues $(3,4,6,7)$, whereas B7-H4 protein expression is tightly regulated and shows limited expression in most normal human and murine tissues $(4,6,8)$. B7-H4 is overexpressed in multiple types and stages of cancer including ovarian, uterine, and endometrial cancers $(6,9)$. The B7-H4 receptor has not been identified; however, evidence suggests that a receptor could be induced and function on $\mathrm{T}$ cells $(3,4)$.

B7-H4 engagement to its receptor on $\mathrm{T}$ cells limits proliferation, cytokine production, and cytotoxicity (3-5). The immunosuppressive and tissue protective function of B7-H4 has been demonstrated in multiple in vivo models. Transgenic B7-H4 overexpression in pancreatic islets protected mice from the development of diabetes mellitus in the presence of islet-specific $\mathrm{T}$ cell response $(7,10)$. Transplantation of islets with B7-H4 overexpression prolonged islet survival (11), and delivery of a B7-H4-Ig fusion protein protected mice against ConA-induced hepatic injury (12). B7-H4-Ig treatment can directly modulate $\mathrm{CD}^{+} \mathrm{T}$ cell inflammatory function (13-15). Conversely, loss of B7-H4 expression on islet cells in autoimmune mice prone to diabetes mellitus accelerated disease progression, whereas pharmacological or genetic approaches that reduce the loss of B7-H4 delayed the onset of diabetes mellitus (16). Host B7-H4 deficiency conferred resistance to a lethal pulmonary infection with Streptococcus pneumoniae (17) or Listeria monocytogenes (18). Collectively, these findings suggest B7-H4 expression on target cells negatively regulates immune function in multiple disease models. B7-H4 overexpression in human tumor tissues (19) and soluble B7-H4 in type 1 diabetes mellitus patient sera (20) support the important role of B7-H4 in human disease progression.

Despite the importance of $\mathrm{B} 7-\mathrm{H} 4$ in peripheral tolerance, $\mathrm{B} 7-\mathrm{H} 4: \mathrm{B} 7-\mathrm{H} 4$ receptor interactions in regulating GVHD have not been studied in detail. Here, we investigated the functional significance of B7-H4 expressed on host tissues and explored the role of B7-H4 expressed on donor T cells in regulating murine acute GVHD. Our findings suggest that both host and donor B7-H4 can downregulate $\mathrm{T}$ cell function during GVHD. We also explored mechanistic underpinnings that contributed to B7-H4-mediated acute GVHD regulation.

\section{Results}

Absence of host B7-H4 expression accelerates GVHD-induced lethality. Although B7-H4 mRNA expression has been detected at low levels in a wide variety of non-lymphoid tissues in healthy persons $(4,6), \mathrm{B} 7-\mathrm{H} 4$ protein expression is more limited because of tight translational control in human and murine peripheral tissues $(4,6,8,21)$. To assess B7-H4 mRNA expression in acute GVHD target tissues, lethally irradiated WT BALB/c $\left(\mathrm{H}-2^{\mathrm{d}}\right)$ recipients were given WT B6 $\left(\mathrm{H}-2^{\mathrm{b}}\right)$ BM with or without purified donor T cells. GVHD organs (spleen, lung, liver, colon, and ileum) were harvested on day 7 and B7-H4 mRNA expression was quantified by qRT-PCR. Compared with mice receiving BM only, recipient mice with WT donor T cells had significantly higher B7-H4 mRNA in the spleen $(P<0.0001)$ and lung $(P<0.0001)$ with a statistical trend $(P=0.06)$ toward higher levels seen in the ileum of GVHD versus naive controls (data not shown).

To determine the physiological significance of host B7-H4 expression in acute GVHD, WT BALB/c or $\mathrm{B} 7-\mathrm{H}_{4}^{-/-}$recipients were given allogeneic WT B6 BM with or without purified T cells. GVHD-induced lethality was significantly accelerated in $\mathrm{B} 7-\mathrm{H} 4^{-/}$recipients compared with WT recipients (Figure $1 \mathrm{~A}$, median survival time [MST], 21.5 days versus 49.5 days; $P<0.0001)$ along with increased clinical GVHD scores (Figure 1B) and accelerated weight loss (Figure 1C). GVHD-induced lethality was accelerated further when B7-H4 ${ }^{-/}$versus WT recipients were given a 2-fold higher T cell dose (Supplemental Figure 1, A-C; supplemental material available online with this article; https://doi.org/10.1172/jci.insight.127716DS1). These data suggest that B7-H4 expression on host tissues can regulate GVHD lethality.

The small frequency $(5 \%-10 \%)$ of $\mathrm{CD} 25^{+}$regulatory $\mathrm{T}$ cells (Tregs) present in the donor graft is sufficient to suppress GVHD-induced lethality (22). A possible explanation for heightened GVHD might be related to diminished expansion or suppressor function of Tregs contained in the donor $\mathrm{T}$ cell graft. Therefore, studies were performed to determine whether CD25-depleted donor grafts given to B7-H4 ${ }^{-/}$versus WT recipients would accelerate GVHD lethality. As expected, CD25-replete T cell grafts caused accelerated lethality in $\mathrm{B} 7-\mathrm{H} 4^{-/-}$versus WT recipients (Figure 1D, $P<0.0001$ ). CD25-depleted T cell grafts caused more rapid lethality in WT recipients $(P=0.016)$. Because CD25-depleted T cells also resulted in the most rapid lethality when infused into $\mathrm{B} 7-\mathrm{H}_{4}{ }^{-/}$versus WT recipients $(P<0.0001)$, these data indicate that host B7-H4 expression-mediated GVHD inhibition does not require Tregs present in the donor graft. 
A

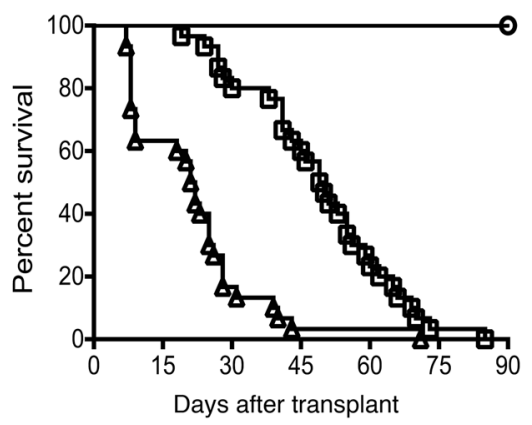

C

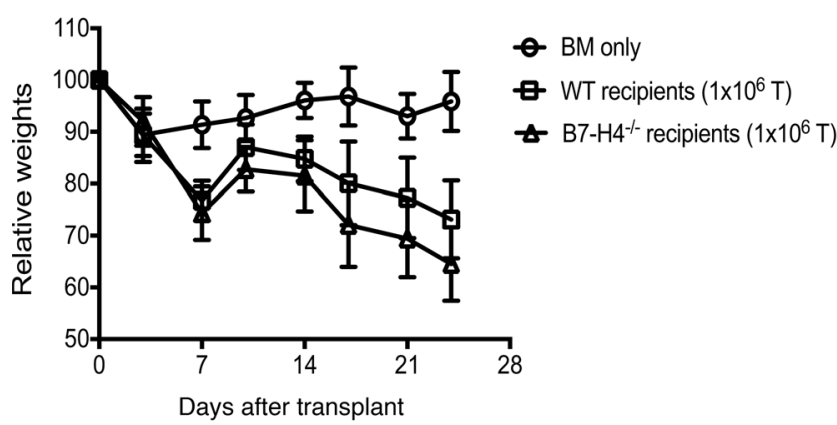

B

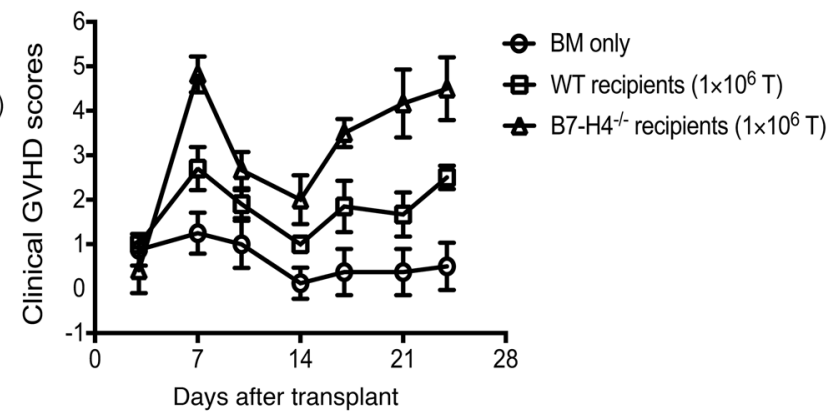

D

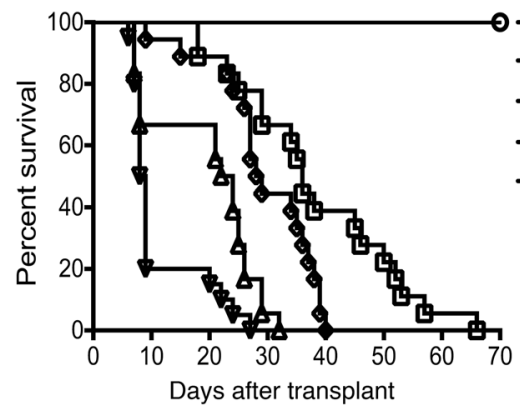

E

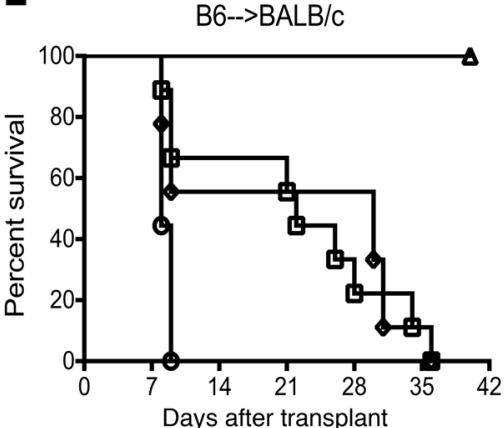

$2 \times 10^{6}$ T cells

- 4 B6-->BALB/c-BALB/C (BM only)

$86-->B A L B / c-B A L B / c$

- B6-->B7-H4KO-BALB/C

- B6->BALB/C-B7-H4KO

Figure 1. Absence of host B7-H4 expression accelerates CVHD lethality and B7-H4 expression on hematopoietic cells is critical for controlling acute GVHD. (A-C) Lethally irradiated WT BALB/c recipients or B7- $4^{-/-}$recipients were infused with $10^{7}$ WT B6 BM cells alone or with $1 \times 10^{6}$ WT B6 purified T cells. (A) Kaplan-Meier survival plot represents pooled data ( $n=21-30$ mice/group) from 3 independent experiments (BM + T cells: WT versus B7-H4 $4^{-/-}$recipients; $\left.P<0.0001\right)$. (B) Transplanted mice were evaluated for clinical GVHD $(n=8-12 /$ group $)$. BM + T cells: WT versus B7-H4 ${ }^{-/-}$ recipients, $P<0.0001$ on d7, d14, d17, d21, and d24; $P=0.0009$ on d10. Data are representative of 3 independent experiments. (C) Relative weights of transplanted mice. Pooled data ( $n=16-22 /$ group) from 2 independent experiments (BM + T cells: WT versus B7-H4 ${ }^{-/-}$recipients; $P<0.05$ on d10, $\mathrm{d} 17, \mathrm{~d} 21$, and d24. (D) Lethally irradiated WT BALB/c recipients or B7- $\mathrm{H}^{-1-}$ recipients were infused with $10^{7}$ WT B6 BM cells alone ( $n=12$ mice) or with $1 \times 10^{6}$ WT B6 purified T cells ( $n=18$ mice/group) or with $1 \times 10^{6}$ WT B6 CD25-depleted purified T cells $(n=18-20$ mice/group). Kaplan-Meier survival plot represents pooled data from 2 independent experiments $\left(\mathrm{BM}+\mathrm{T}\right.$ cells: WT versus B7-H4 ${ }^{-1-}$ recipients, $P<0.0001 ; \mathrm{BM}+\mathrm{CD} 25-\mathrm{depleted}$ T cells: WT versus $B 7-H 4^{-1-}$ recipients, $P<0.0001$; WT recipients: $B M+T$ cells versus $B M+C D 25-$ depleted T cells, $P=0.016 ; B 7-H^{-1-}$ recipients: $B M$ $+T$ cells versus $B M+C D 25$-depleted T cells, $P=0.008$. (E) Lethally irradiated WT BALB/c recipients or B7-H4-/- recipients were infused with BM cells from B7- $4^{-/-}$or WT BALB/c mice, respectively, to create chimeras. We also created control chimeras (WT $\rightarrow$ WT). After 3 months, these chimeras were re-irradiated and infused with allogeneic WT B6 BM cells with $2 \times 10^{6}$ WT B6 purified T cells. Kaplan-Meier survival plot of transplanted mice ( $n=8-9 /$ group) is shown. WT $\rightarrow$ WT versus B7-H4 ${ }^{-1-} \rightarrow$ WT chimeras, $P=0.0028 ; \mathrm{WT} \rightarrow \mathrm{B} 7-\mathrm{H} 4^{-/-}$versus $\mathrm{B} 7-\mathrm{H} 4^{-/-} \rightarrow \mathrm{WT}$ chimeras, $P=0.0141$. Data are representative of 2 independent experiments. (B and $\mathbf{C}$ ) Data represent mean \pm SEM. $P$ values were calculated by 2 -tailed $t$ test $(\mathbf{B}$ and $\mathbf{C})$ or logrank test (A, D, and $\mathbf{E})$. 
B7-H4 expression on hematopoietic cells is more critical than parenchymal cells for controlling acute GVHD. B7$\mathrm{H} 4$ is expressed on both hematopoietic and parenchymal cells. To determine whether B7-H4 expression on hematopoietic and parenchymal cells are equally important in regulating GVHD, we created BM chimeras by lethal radiation conditioning of the host. Prior to induction of GVHD, syngeneic chimeras were generated to fix B7-H4 loss to either hematopoietic or parenchymal cells: B7-H4 ${ }^{-/} \rightarrow$ WT in which tissue cells were $\mathrm{B} 7-\mathrm{H}_{4}^{+/+}$and BM-derived cells were $\mathrm{B} 7-\mathrm{H} 4^{-/-} ; \mathrm{WT} \rightarrow \mathrm{B} 7-\mathrm{H} 4^{-/-}$chimeras in which tissue cells were $\mathrm{B} 7-\mathrm{H}_{4}{ }^{--}$and BM-derived cells were $\mathrm{B} 7-\mathrm{H} 4^{+/+}$; and control chimeras (WT $\rightarrow \mathrm{WT}$ ). After 3 months, peripheral blood lymphocytes (PBLs) and spleens were analyzed for engraftment; nearly $98 \%$ of antigen-presenting cells were of donor BM origin (PBLs: $97.2 \pm 0.96$; spleens: $90.6 \pm 0.83$; data not shown). Chimeras were re-irradiated and infused with allogeneic WT B6 BM plus T cells $\left(2 \times 10^{6}\right)$ to induce GVHD. B7-H4 $4^{-1-} \rightarrow$ WT chimeras died significantly faster than $\mathrm{WT} \rightarrow \mathrm{WT}$ and $\mathrm{WT} \rightarrow \mathrm{B} 7-\mathrm{H}_{4}^{-{ }^{-}}$chimeras (Figure $1 \mathrm{E}, P=0.0028$ and $P=0.014$, respectively). However, $\mathrm{WT} \rightarrow \mathrm{B} 7-\mathrm{H} 4^{-/}$survival did not differ from $\mathrm{WT} \rightarrow \mathrm{WT}$ chimeras $(P=$ 0.799), suggesting that B7-H4 expression on hematopoietic cells is critical for dampening GVHD lethality.

At a lower T cell dose $\left(1 \times 10^{6}\right), \mathrm{B} 7-\mathrm{H}^{-/-} \rightarrow \mathrm{WT}$ chimeras again died significantly faster than WT $\rightarrow$ WT chimeras (Supplemental Figure 1D, $P=0.0287$ ). A statistical trend toward faster lethality was seen in $\mathrm{WT} \rightarrow \mathrm{B} 7-\mathrm{H} 4^{-/-}$versus $\mathrm{WT} \rightarrow \mathrm{WT}$ chimeras $(P=0.078)$ but in contrast to the higher $\mathrm{T}$ cell dose, survival of $\mathrm{B} 7-\mathrm{H} 4^{-1-} \rightarrow \mathrm{WT}$ chimeras did not differ from WTB7-H4 ${ }^{-{ }^{-}}$chimeras $(P=0.915)$. Taken together, these data show a dominant effect of B7-H4 expression on hematopoietic cells in regulating GVHD with a less pronounced and variable effect of parenchymal B7-H4 expression.

Absence of B7-H4 expression on host cells increases donor T cell proliferation capacity and effector function. Increased donor $\mathrm{T}$ cell expansion in $\mathrm{B} 7-\mathrm{H} 4^{-/}$recipients may drive accelerated GVHD lethality. B6 LUC transgenic $\left(\mathrm{LUC}^{+}\right) \mathrm{T}$ cells given to WT or B7-H4 ${ }^{-/-}$recipients produced a significantly higher LUC signal intensity by day 7 after bone marrow transplant (BMT) (Supplemental Figure 2A). Because conditioning-induced intestinal tract damage plays a primal role in acute GVHD, we analyzed the expression of gut adhesion and chemokine homing receptors $(23,24)$ as well as cell activation markers. Day 7 after BMT splenic donor T cells in B7- $\mathrm{H} 4^{-/-}$recipients had evidence of activation $\left(\mathrm{CD} 44^{\mathrm{hi}}, \mathrm{CD} 25^{+}\right.$, and $\left.\mathrm{CD} 62 \mathrm{~L}^{\mathrm{lo}}\right)$ and increased proliferation by Ki-67 staining (Supplemental Figure 2, B-E). The percentage of donor $\mathrm{CD} 4^{+}$and $\mathrm{CD}^{+} \mathrm{T}$ cells expressing gut-homing receptors (CXCR3, LPAM-1, CCR5, and CCR9) (Supplemental Figure 2, F-I) or proinflammatory cytokines (IFN- $\gamma$, TNF- $\alpha$, IFN- $\gamma / \mathrm{TNF}-\alpha$, IFN- $\gamma / \mathrm{CD} 107 \mathrm{a}, \mathrm{IFN}-\gamma / \mathrm{LPAM} 1$, or IL-22) was significantly increased $(P<0.05)$ in $\mathrm{B} 7-\mathrm{H} 4^{-/-}$versus WT recipients (data not shown).

Increased donor $\mathrm{CD}^{+}$and $\mathrm{CD}^{+} \mathrm{T}$ cell numbers in the spleen (Figure 2A) reflected higher proliferation capacity (Figure 2B) and responder frequency (Supplemental Figure 3A), along with lower apoptosis (annexin V, FasL) (Figure 2, C and D) and reduced expression of coinhibitory molecules (Supplemental Figure 4) in B7-H4 ${ }^{-/}$versus WT recipients. Donor T cell analysis in mesenteric lymph nodes also reflected increased proliferation and lower apoptosis in $\mathrm{B} 7-\mathrm{H} 4^{-/-}$recipients (Supplemental Figure 3 , E-H). CD127 (the IL-7R $\alpha$ chain that transduces cell survival signals) expression on donor T cells was significantly higher in B7- $\mathrm{H}^{-/-}$versus WT recipients (Figure 2E and Supplemental Figure 3B). Thus, host B7-H4 deficiency failed to restrain donor T cell expansion, likely contributing to GVHD lethality. Histological analysis on day 21 showed increased spleen and colon injury in B7-H4 $4^{-/}$versus WT recipients (Figure 3A) and loss of epithelial integrity (Figure 3B) as measured by higher levels of orally administered FITC-dextran, detectable in peripheral blood if the epithelial barrier has been compromised (25). Donor $\mathrm{CD}^{+}$and $\mathrm{CD}^{+} \mathrm{T}$ cell numbers in colon lamina propria were increased in $\mathrm{B} 7-\mathrm{H} 4^{-/-}$recipients along with increased expression of $\mathrm{Ki}-67$, homing receptors, cytolytic pathway antigen expression, and proinflammatory cytokines (Figure 3, C-K, and Supplemental Figure 5).

Allogeneic donor $\mathrm{T}$ cells infused into $\mathrm{B} 7-\mathrm{H}^{-/-}$versus $\mathrm{WT}$ recipients require multiple substrate transporters and metabolic pathways to meet high metabolic demands. Proliferating donor T cells can increase TCA cycle activity and NADH (driving ROS) production to hyperpolarize mitochondrial membrane potential $(\Delta \Psi \mathrm{m})$. Mitochondrial activity of donor T cells was measured with tetramethylrhodamine (TMRM), a dye that accumulates within mitochondria in proportion to $\triangle \Psi \mathrm{m}$. TMRM fluorescence intensity was significantly higher in B7-H4 ${ }^{-/}$versus WT recipients (Figure $2 \mathrm{~F}$ and Supplemental Figure $3 \mathrm{C}$ ) along with increased superoxide production by activated donor $\mathrm{T}$ cells (Figure $2 \mathrm{G}$ ) because higher $\Delta \Psi \mathrm{m}$ could induce superoxide production from mitochondrial respiratory chains. Notably, donor T cells in B7-H4 ${ }^{-/-}$recipients had more mitochondrial mass and respiring mitochondria, as determined by staining with mitotracker green (MTG) and mitotracker deep red (MTDR), respectively (Figure 2H and Supplemental Figure 3D). 
A

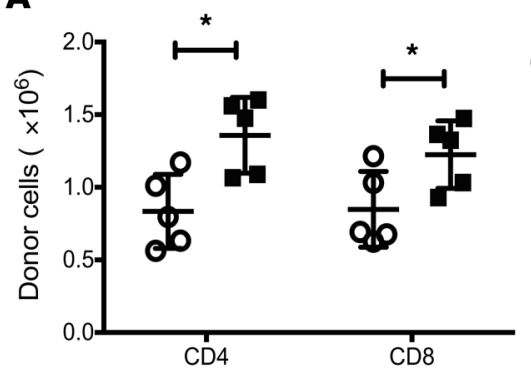

B

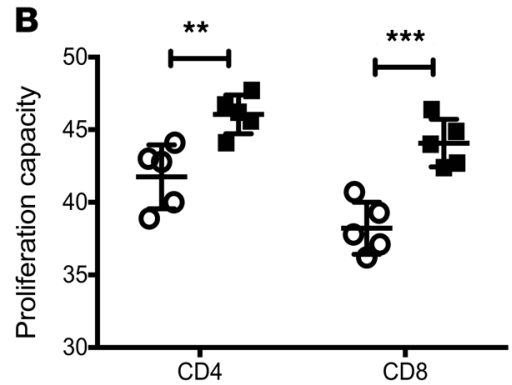

C

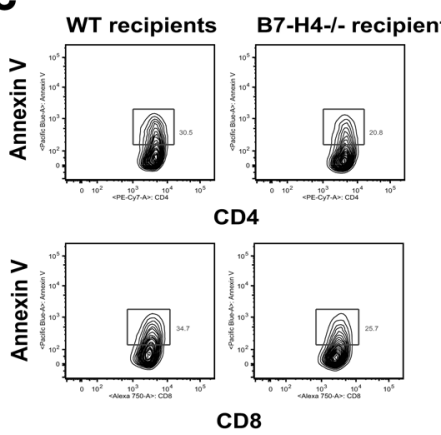

E

E WT recipients B7-H4-/- recipients
- WT recipients

B7- $-4^{-/-}$recipients

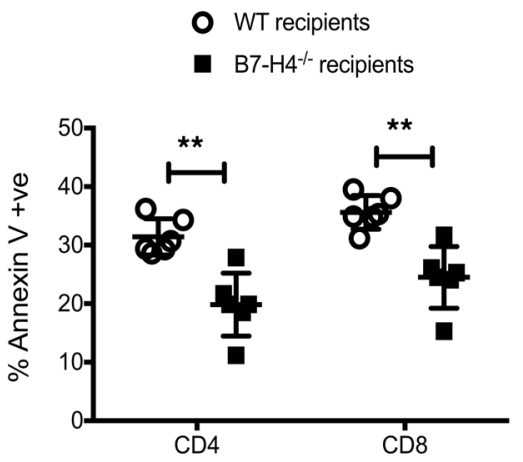

D

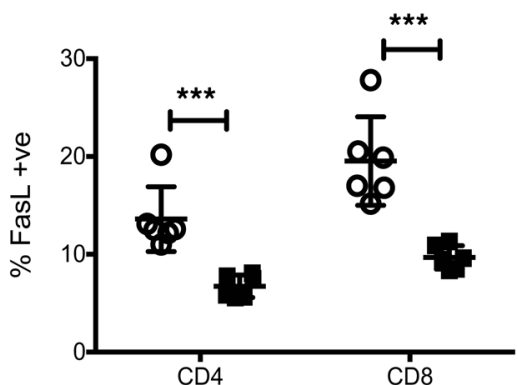

CD4

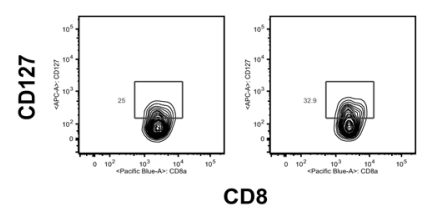

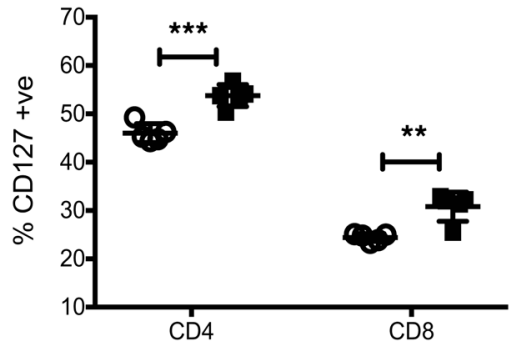

$\mathbf{F}$

- WT recipients

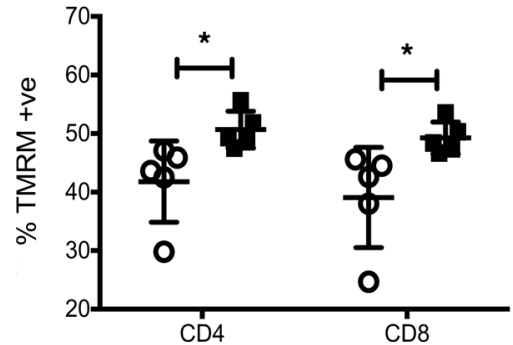

G

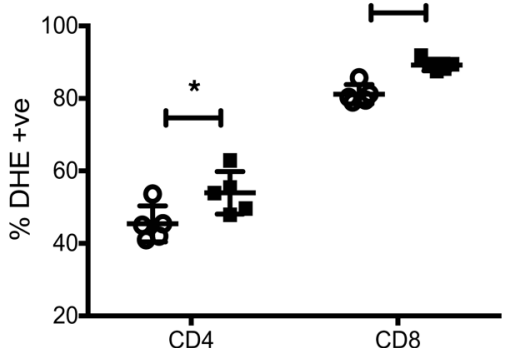

H

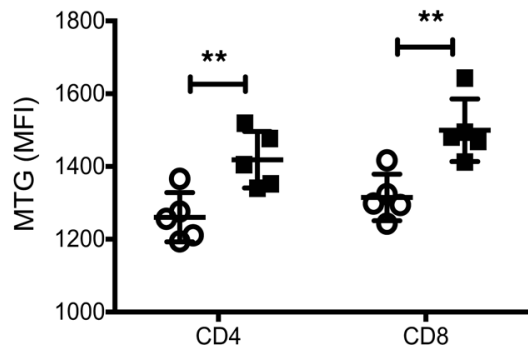

Figure 2. Absence of B7-H4 expression on host cells increases proliferation and survival of allogeneic donor T cells. Lethally irradiated WT BALB/c recipients or B7- $4^{-/-}$recipients were infused with $10^{7}$ WT B6 BM cells plus $20 \times 10^{6} \mathrm{CFSE}$-labeled or $20 \times 10^{6}$ CTV-labeled WT B6 splenocytes. Mice were sacrificed on day 4 after BMT, and splenocytes were analyzed by flow cytometry. Donor T cells were analyzed for total cell numbers (A), CFSE dilution (B), Annexin V (C), FasL (D), TMRM (F), DHE (C), or MTC (H) expression. (E) Lethally irradiated WT BALB/c recipients or B7-H4 $4^{-/-}$recipients were infused with $10^{7}$ WT B6 BM cells plus $2 \times 10^{6}$ WT B6 purified T cells. Splenocytes were analyzed on day 4 to detect the percentage of donor T cells expressing CD127. (A-H) Data are representative of at least 5 mice per group from 2-3 independent experiments. MFI, mean fluorescence intensity. Data represent mean $\pm \mathrm{SEM}$, and $P$ values were calculated by 2-tailed $t$ test. ${ }^{*} P<0.05 ;{ }^{* *} P<0.01 ;{ }^{* *} P<0.001$.

Increased donor $\mathrm{T}$ effector proliferation, $\Delta \Psi \mathrm{m}$, and mitochondrial mass prompted functional metabolic analysis because distinct $\mathrm{T}$ cell metabolism can determine both $\mathrm{T}$ cell fate and function. To fulfill the bioenergetic and biosynthetic demands of proliferation, activation, migration, and cytolysis, T cells reprogram their metabolic pathways to use different substrates, including glucose, glutamine, and fatty acids (FAs) (26). Activated T cells 
A

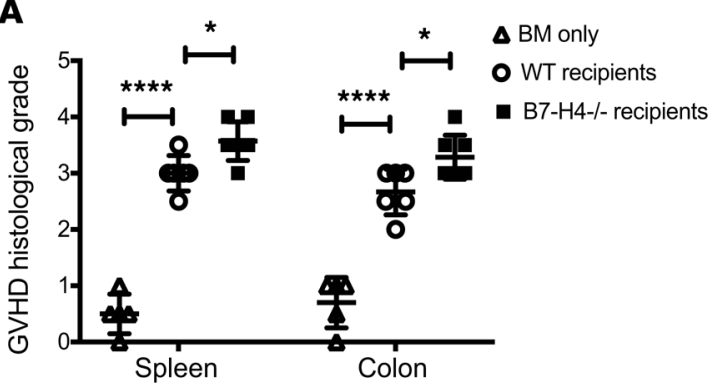

C

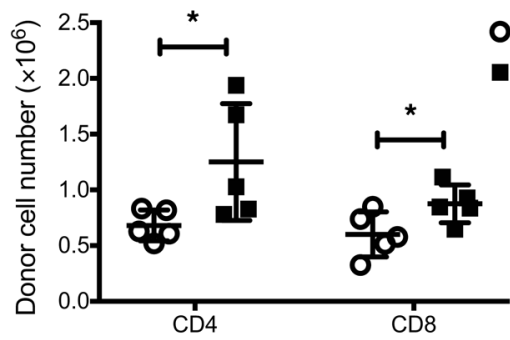

F

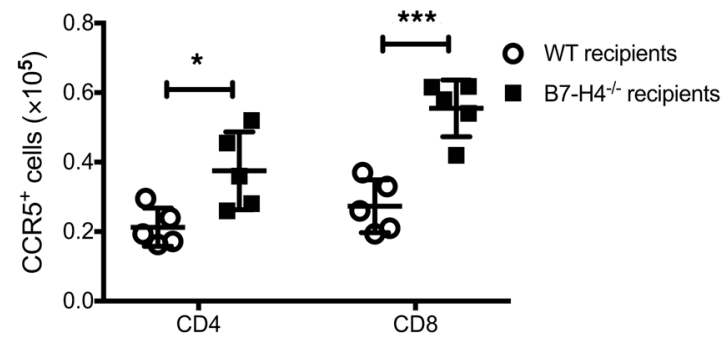

I

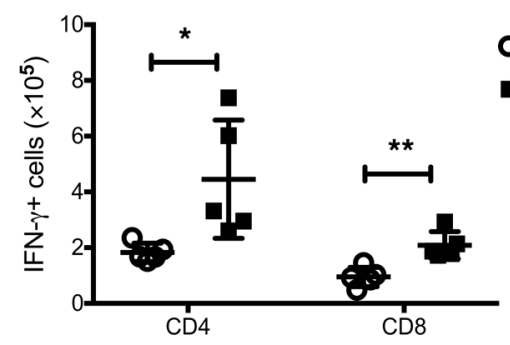

B

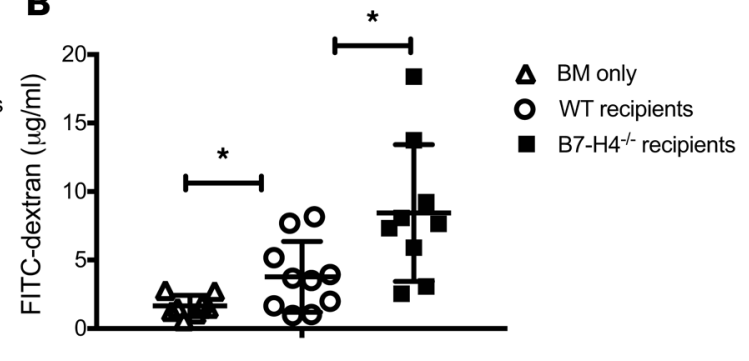

D

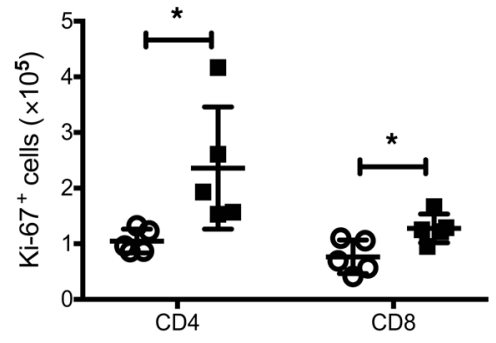

E

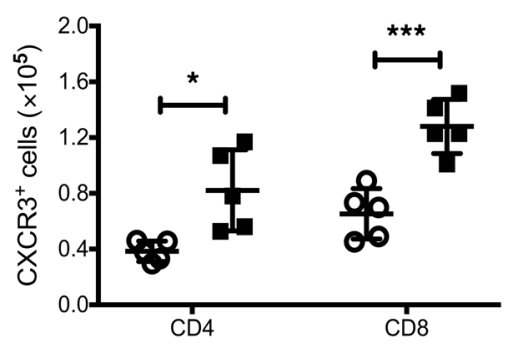

H
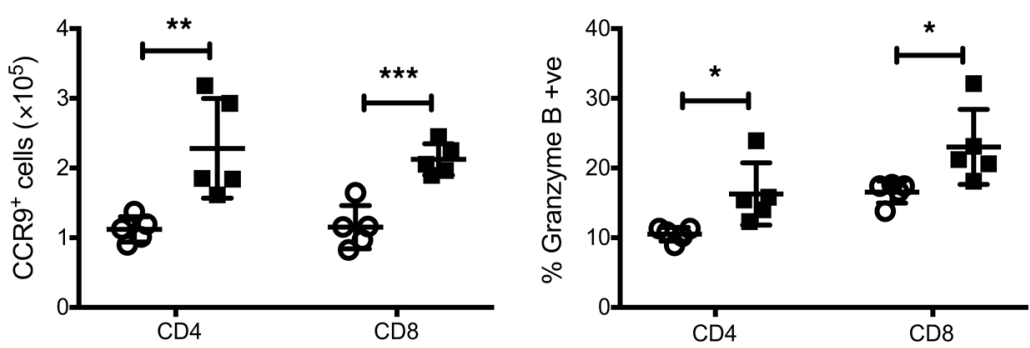

J

\section{K}

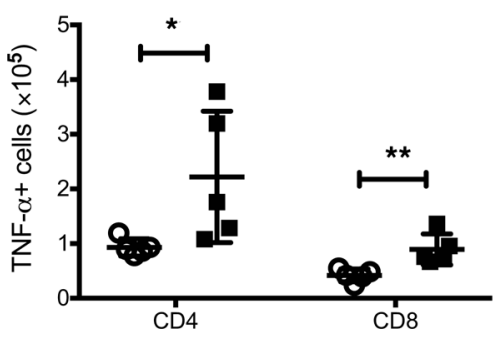

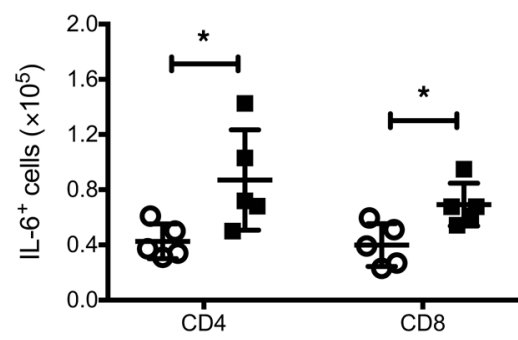

Figure 3. Absence of B7-H4 expression on host cells increases donor T cell effector function that promotes increased gut injury in recipients. Lethally irradiated WT BALB/c recipients or B7-H4 ${ }^{-/-}$recipients were infused with $10^{7}$ WT B6 BM cells alone or with $1 \times 10^{6}$ WT B6 purified T cells. (A) Mice were sacrificed ( $n=5-7 /$ group) on day 21 after BMT, and H\&E-stained tissue sections were scored for GVHD. (B) FITC-dextran was administered orally on day 22 after BMT ( $n=8-10$ mice/group), and plasma levels were measured after 4 hours. (C-K) Mice were sacrificed on day 23 after BMT ( $n=10 / g r o u p)$, and lymphocytes isolated from the colon ( 2 colons were pooled to make 1 pooled sample and 5 pooled samples per group) were analyzed by flow cytometry. Donor $\mathrm{CD}^{+}$and CD8 ${ }^{+} \mathrm{T}$ cells were analyzed for total cell numbers (C), and surface expression of CXCR3 (E), CCR5 (F), or CCR9 (C). Donor T cells were also analyzed for intracellular expression of Ki-67 (D), Granzyme B (H), IFN- $\gamma$ (I), TNF- $\alpha$ (J), or IL-6 (K). (A-K) Data are representative of 2 independent experiments. Data represent mean \pm SEM. $P$ values were calculated by 2 -tailed $t$ test. ${ }^{*} P<0.05 ;{ }^{* *} P<0.01 ;{ }^{* *} P<0.001 ;{ }^{* * *} P<0.0001$.

preferentially generate ATP through aerobic glycolysis (27). GLUT1, the major glucose transporter on hematopoietic cells (28), was significantly higher on proliferating donor T cells from B7-H4 ${ }^{-/}$versus WT recipients (Figure 4A). Seahorse measurements showed an increased extra-cellular acidification rate (ECAR) following exposure to glucose, and maximal glycolytic capacity (after adding oligomycin to suppress mitochondrial ATP production) of donor $\mathrm{T}$ cells isolated from $\mathrm{B} 7-\mathrm{H}_{4}^{-/-}$recipients (Figure $4 \mathrm{~B}$ and Supplemental Figure $6 \mathrm{~A}$ ).

Oxidative metabolism can use glucose, glutamine, or FAs as substrates. Both the basal oxygen consumption rate (OCR; an indicator of oxidative phosphorylation) and maximal respiratory capacity 
were significantly elevated on donor T cells isolated from B7- $\mathrm{H} 4^{-/-}$versus WT recipients (Figure 4 , C and D). Glutamine is a key amino acid for $\mathrm{T}$ cell activation and an amine group donor for nucleotide synthesis. Glutamate, the first product of glutamine metabolism, can be converted into $\alpha$-ketoglutarate and metabolized through the TCA cycle to generate citrate, providing a 2-step pathway for glutamine to enter energy metabolism. Cell surface expression of CD98 (glutamine transporter) on donor T cells also was significantly higher in $\mathrm{B} 7-\mathrm{H}_{4}{ }^{-/-}$recipients (Supplemental Figure $6 \mathrm{~B}$ ). Iron, an essential micronutrient for cell growth, catalyzes key reactions in energy metabolism and DNA synthesis. Transferrin transports iron from sites of absorption and storage to sites of iron utilization. Iron-bound transferrin interacts with transferrin receptor to promote iron transport across the cell membrane. Cell surface expression of CD71 (transferrin receptor) on donor T cells was significantly higher in $\mathrm{B} 7-\mathrm{H} 4^{-/-}$recipients (Supplemental Figure 6C).

GVHD T effectors are under high metabolic stress $(29,30)$ and can harness glycolysis, glutaminolysis, and fatty acid oxidation (FAO) pathways to provide supplemental energy for survival and/or function $(26,31)$. FA uptake as quantified using BoDipy ${ }_{\mathrm{C} 1-\mathrm{C} 12}$, a fluorescent $\mathrm{FA}$ analog, and surface expression of CD36, a receptor that binds long-chain FAs (32), were significantly elevated on donor $\mathrm{T}$ cells obtained from $\mathrm{B} 7-\mathrm{H}_{4}{ }^{-/}$recipients (Figure $4, \mathrm{E}$ and $\mathrm{F}$ ). CPT1a, the rate-limiting enzyme in mitochondrial FAO, was significantly higher on proliferating donor T cells from B7-H4 $4^{-/-}$versus WT recipients (Figure $4 \mathrm{G}$ and Supplemental Figure 6D). Etomoxir, a CPT1a inhibitor, decreased the maximal respiratory capacity of donor T cells obtained from B7-H4 ${ }^{-/-}$versus WT recipients (Figure 4, H and I, and Supplemental Figure 6E), confirming higher mitochondrial FAO in $\mathrm{B} 7-\mathrm{H} 4^{-/-}$recipients. Metabolic pathway analysis of donor $\mathrm{T}$ cells isolated from the colon on day 23 after BMT also suggested elevated expression of GLUT1, CD98, CPT1a, and CD71 in B7-H4 ${ }^{-/-}$versus WT recipients (Supplemental Figure 7).

B7-H4 deficient donor $T$ cells cause increased GVHD lethality that is independent of donor Treg function. Moderate B7-H4 expression was detected on tumor-infiltrating CD8 T cells in E.G7-tumor-bearing mice (33). B7-H4 expression could be induced on human T cells, B cells, dendritic cells, and monocytes after in vitro stimulation with mitogens (LPS, PHA, PMA, or ionomycin) (4). Therefore, B7-H4 expression on the cell surface appears to be activation-induced and its expression on $\mathrm{T}$ cells has not been analyzed in the context of GVHD. To perform the analysis, lethally irradiated B6 or BALB/c recipients were infused with WT B6 BM and B6 congenic (CD45.1) T cells to induce GVHD, or lethally irradiated $\mathrm{BALB} / \mathrm{c}$ or B6 recipients were infused with WT BALB/c BM and BALB/c congenic (CD45.1) T cells. In both allogeneic strain combinations, B7-H4 expression was significantly elevated on donor CD4 ${ }^{+}$and $\mathrm{CD}^{+} \mathrm{T}$ cells compared with syngeneic donor $\mathrm{T}$ cells (Figure 5, A and B, and Supplemental Figure 8A). These experiments confirmed a marked upregulation of B7-H4 expression on alloreactive donor T cells under GVHD conditions. To determine whether anti-B7-H4 fluorochrome cross-reactivity accounted for these findings, allogeneic WT BM and B7- $4^{-/-}$versus WT BALB/c donor T cells were infused into B6 recipients. B7-H4 fluorochrome binding was detected on less than $3 \%$ of syngeneic WT or $1 \%$ or less of allogeneic $\mathrm{B} 7-\mathrm{H}^{-/-} \mathrm{CD}^{+}$or $\mathrm{CD}^{+} \mathrm{T}$ cells, in contrast with mean values in allogeneic WT $\mathrm{T}$ cells ( $15 \%$ or $20 \%$, respectively; $P<0.0001$ ) noted to have maximum expression on day 5 after BMT (Figure $5 \mathrm{C}$ and Supplemental Figure $8 \mathrm{~B}$ ). Increased activation and effector function of $\mathrm{B} 7-\mathrm{H} 4^{+}$compared with B7-H4- WT donor T cell population is marked by elevated CD25, CD44, CD69, granzyme B, and perforin, or reduced CD62L expression (Supplemental Figure 8, C-H).

To determine whether B7-H4 expression on donor T cells affects GVHD, lethally irradiated WT B6 recipients were infused with WT BALB/c BM with or without purified T cells from WT BALB/c or B7-H4 ${ }^{-/-}$ donors. GVHD-induced lethality was markedly accelerated when recipients were given $\mathrm{B} 7-\mathrm{H} 4^{-/-}$versus WT donor $\mathrm{T}$ cells at a dose of $3 \times 10^{6}$ (MST, 9 days versus 49 days; $P<0.0001$, Supplemental Figure 8I). GVHD-induced lethality also was accelerated in recipients of $\mathrm{B} 7-\mathrm{H}_{4}{ }^{-/-}$versus WT donor $\mathrm{T}$ cells at a lower dose of $2 \times 10^{6}$ (MST, 39 days versus 66 days; $P<0.0001$, Figure 5D) along with increased clinical GVHD scores (Figure 5E) and heightened weight loss (Supplemental Figure 8J).

To determine whether hypofunctional Tregs were responsible for accelerated GVHD $(22,34)$, B7-H4 ${ }^{-\prime}$ versus WT Tregs were added to responder $\mathrm{T}$ cells and anti-CD3 $\mathrm{mAb}$-driven $\mathrm{T}$ cell proliferation was quantified. B7-H4 ${ }^{-/}$versus WT Tregs were similarly effective (Supplemental Figure 9). In addition, accelerated GVHD lethality in recipients of B7-H4 $4^{-/}$versus WT donor T cells was independent of donor Treg function because Treg depletion from donor T cell graft did not abrogate GVHD acceleration $(P<0.0001$, Figure $5 \mathrm{~F})$. Moreover, in vivo, in a system in which supplementary donor Tregs were used to mitigate GVHD, B7-H4 ${ }^{-/}$ 
A

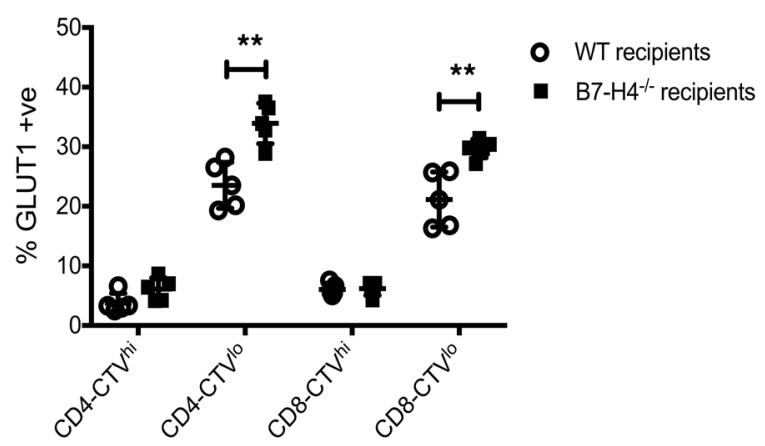

C

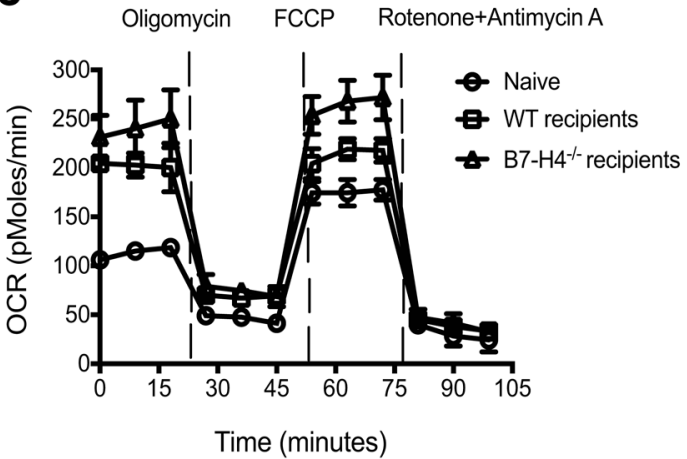

B

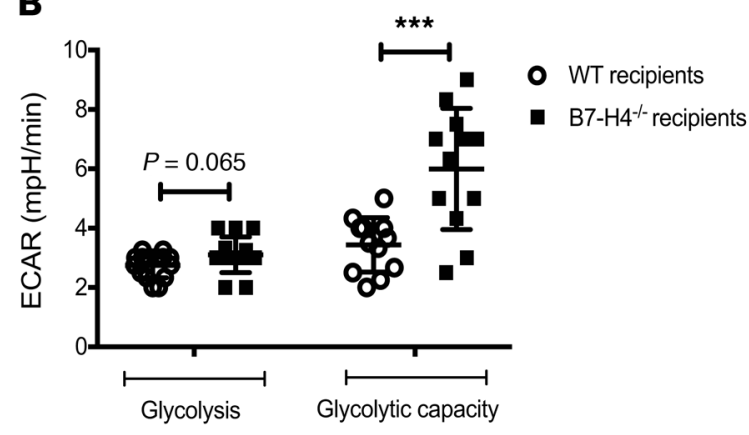

D

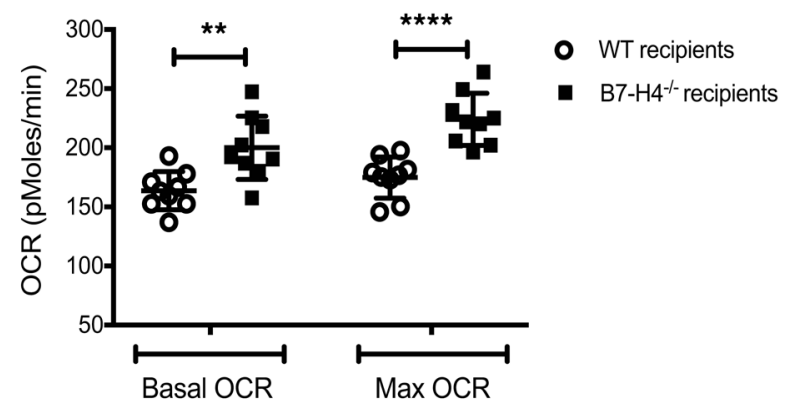

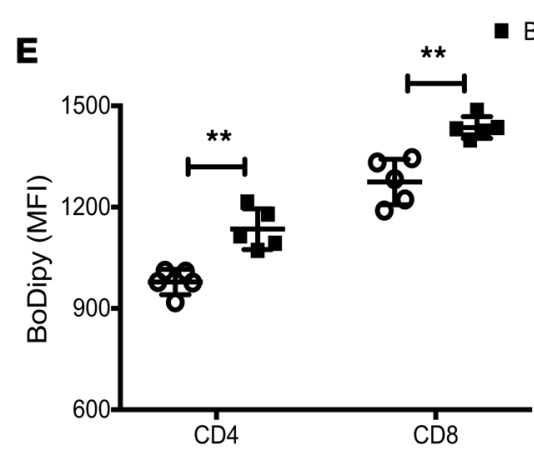

H

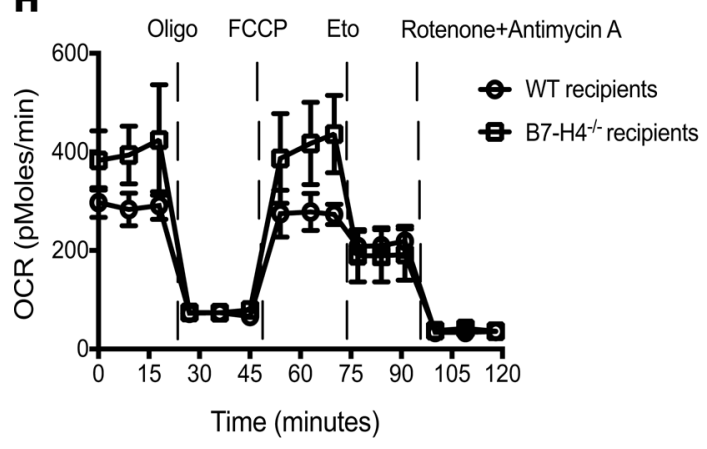

- WT recipients

- B7-H4--- recipients

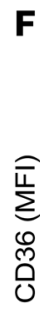<smiles>[CH]C=[Co]</smiles>

- WT recipients

- B7- $4^{-1-}$ recipients

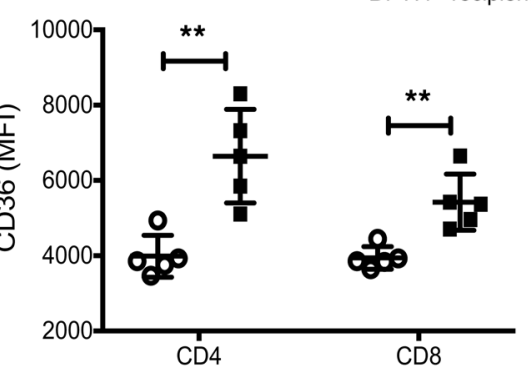

I

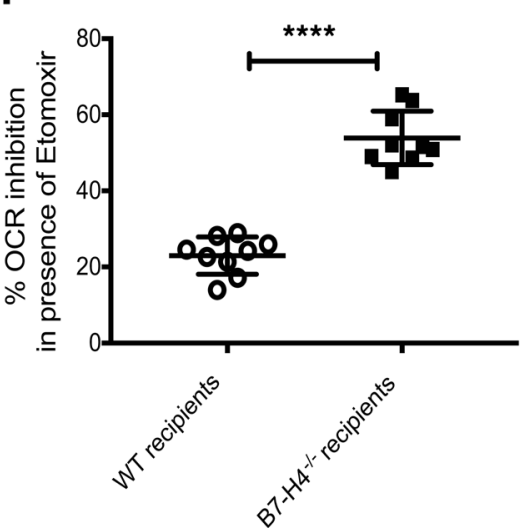

- WT recipients

G

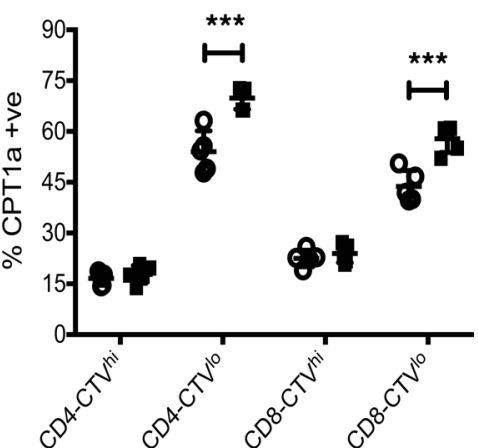

Figure 4. Metabolic alterations of donor T cells in WT versus B7-H4-/- recipients. Lethally irradiated WT BALB/C recipients or B7-H4-/- recipients were infused with $10^{7}$ WT B6 BM cells plus $20 \times 10^{6}$ CTV-labeled or unlabeled WT B6 splenocytes. Mice were sacrificed on day 4 after BMT, and experiments were performed as described. (A) Splenocytes were analyzed by flow cytometry for intracellular expression of GLUT1 in undivided (CTVhi) and divided (CTV ${ }^{\circ}$ ) donor T cells. (B) Extra-cellular acidification rate (ECAR) of purified donor T cells was measured after addition of glucose, oligomycin, and 2-deoxyglucose (2-DG). Basal glycolysis was measured after addition of glucose, and glycolytic capacity was measured after addition of oligomycin. The graph represents pooled data from 5 time points to measure glycolysis and pooled data from 4 time points to measure glycolytic capacity. (C) Oxygen consumption rate 
(OCR) of purified donor T cells was measured after addition of oligomycin, fluorocarbonyl cyanide phenylhydrazone (FCCP), and rotenone plus antimycin A. T cells from naive WT B6 mice $(n=4)$ were included as control. (D) Basal OCR (resting OCR - rotenone plus antimycin A OCR) was measured before the addition of oligomycin, and maximal OCR was measured after addition of FCCP subtracting nonmitochondrial OCR (rotenone plus antimycin A OCR). The graph represents pooled data from 3 time points to measure basal OCR or maximal OCR. Splenic donor T cells were analyzed by flow cytometry for BoDipy (E) or CD36 (F) expression. (G) Splenocytes were also analyzed by flow cytometry for intracellular expression of CPT1a in undivided and divided donor T cells. (H and I) OCR of purified donor T cells was measured after addition of oligomycin, FCCP, etomoxir (Eto), and rotenone plus antimycin A. (I) Percent inhibition was calculated using pooled data from 3 time points after addition of etomoxir. (A and $\mathbf{E}-\mathbf{G}$ ) Data are representative of 5 mice per group from 2-3 independent experiments. (B-D, H, and I) Data are representative of 12 mice per group from 2-3 independent experiments. MFI, mean fluorescence intensity. Data represent mean SEM, and $P$ values were calculated by 2 -tailed $t$ test. ${ }^{*} P<0.01 ;{ }^{* *} P<0.001 ;{ }^{* * *} P<0.0001$.

Tregs were at least as effective as WT Tregs in improving survival of animals (Figure 5G), although we cannot exclude a superior function of $\mathrm{B} 7-\mathrm{H}_{4}{ }^{-/}$versus WT Tregs in GVHD protection under more aggressive GVHD conditions. Together, these data indicate that accelerated GVHD seen with B7-H4 ${ }^{-/-}$versus WT donor T cells was not a result of in vivo Treg hypofunction.

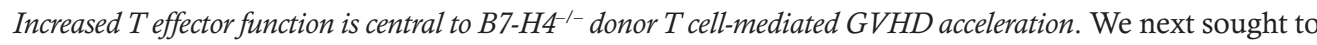
identify potential mechanism(s) for accelerated GVHD. The absolute numbers, proliferation capacity, and $\mathrm{CD} 127$ expression on donor $\mathrm{CD}^{+}$and $\mathrm{CD}^{+} \mathrm{T}$ cells were significantly higher (Supplemental Figure 10, A, $\mathrm{B}$, and E), whereas expression of apoptotic proteins (Supplemental Figure 10, C and D) and coinhibitory receptors, including CD73 and 2B4, were significantly lower $(P<0.05)$ in recipients given $\mathrm{B} 7-\mathrm{H} 4^{-/-}$versus WT donor T cells (data not shown).

B7-H4 $4^{-/-}$versus WT donor T cells had evidence of activation (CD44 ${ }^{\mathrm{hi}}, \mathrm{CD}^{2} 5^{+}$, and CD62L ${ }^{\mathrm{lo}}$ ) (Supplemental Figure 11, A-C), increased expression of gut homing receptors (Supplemental Figure 11, D-G), and the percentage of donor T cells expressing IFN- $\gamma$, coexpressing IFN- $\gamma$ and CD107a, or LPAM-1 was significantly increased on day 7 after BMT (Supplemental Figure 11, H-J). Histological analysis on day 30 after BMT showed increased damage of the colon in recipients given $\mathrm{B} 7-\mathrm{H} 4^{-/-}$donor $\mathrm{T}$ cells (Figure 6A). This phenomenon was associated with increased FITC-dextran levels in peripheral blood (Figure 6B), increased proliferation of donor $\mathrm{T}$ cells (Figure 6C), and increased expression of homing receptors (Figure 6, D and E) and proinflammatory cytokines (Figure 6, F-J) by donor T cells isolated from the colon.

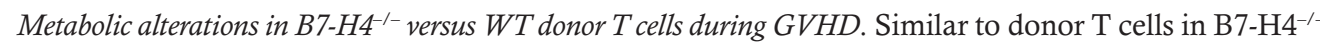
recipients, B7-H4 ${ }^{-/-}$versus WT donor $\mathrm{T}$ cells had increased mitochondrial activity, superoxide production, and respiring mitochondria (Supplemental Figure 10, F-H). Analysis of specific metabolic pathways showed that percent positivity and MFI of GLUT1 expression on proliferating B7-H4 ${ }^{-/}$donor T cells were significantly higher compared with WT donor T cells, suggesting increased glucose uptake and metabolism (Figure 7A, data not shown). Seahorse measurements of the ECAR confirmed increased glucose metabolism by B7-H4 ${ }^{-/-}$donor T cells (Figure 7B). Basal OCR and maximal respiratory capacity were significantly elevated in $\mathrm{B} 7-\mathrm{H} 4^{-/-}$versus WT donor T cells (Figure 7, C and D) as were the percent positive and MFI of CD98 or CD71 expression (data not shown). Increased FA uptake and FAO by B7-H4 ${ }^{-/}$donor T cells were noted (Figure 7, E-G). The use of a specific inhibitor of CPT1a significantly inhibited maximal respiratory capacity of B7-H4 $4^{-/}$versus WT donor T cells (Figure 7, H and I), indicating that mitochondrial FAO was higher in $\mathrm{B} 7-\mathrm{H}_{4}^{-/-}$donor $\mathrm{T}$ cells. In agreement with the data obtained at day 5 after BMT, metabolic pathway analysis of donor T cells isolated from the colon on day 29 after BMT confirmed increased metabolic activities for substrates including glucose, glutamine, FAs, and iron as suggested by elevated expression of GLUT1, CD98, CPT1a, and CD71, respectively, in B7-H4 ${ }^{-/-}$versus WT donor T cells (data not shown).

The graft-versus-leukemia effect is retained in $\mathrm{B7}-\mathrm{H} 4^{-1-}$ recipients of $\mathrm{WT}$ donor $\mathrm{T}$ cells and $\mathrm{WT}$ recipients of $\mathrm{B7}-\mathrm{H} 4^{-1-}$ donor T cells. Elevated B7-H4 expression detected in multiple human cancer types is often associated with poor prognosis (35). Mouse in vivo and human in vitro studies using various anti-B7-H4 antibodies have shown success in rescuing $\mathrm{T}$ cell function in vitro $(3,4,36)$, augmenting $\mathrm{T}$ cell responses in vivo $(3,4)$, and reducing tumor burden in murine lung cancer (37) and the A20 B cell lymphoma (33) models. To assess the graft-versus-leukemia (GVL) response, lethally irradiated WT BALB/c or B7-H4 ${ }^{-1}$ recipients were infused with WT B6 TCD BM and a low dose of T cells $\left(0.5 \times 10^{6}\right)$ plus 3 times the lethal dose of BALB/c recipient strain A20 $0^{\text {luc }-1 y m p h o m a ~ c e l l s ~(38) ~ o n ~ d a y ~} 0$. Mice that received BM and A20 $0^{\text {luc }}$ alone died by day 25 after BMT as a result of tumor burden (Supplemental Figure 12, A-C). WT or B7-H4 ${ }^{-/-}$recipients that received $\mathrm{A} 20^{\text {huc }}$ and donor T cells had no tumor growth (Supplemental Figure 12, A and C) and died from GVHD (Supplemental Figure 12B) that was accelerated in $\mathrm{B} 7-\mathrm{H} 4^{-/-}$versus WT recipients $(P=0.016)$. Thus, the absence of $\mathrm{B} 7-\mathrm{H} 4$ on host hematopoietic and parenchymal cells did not abrogate the GVL effect. 


\section{A}

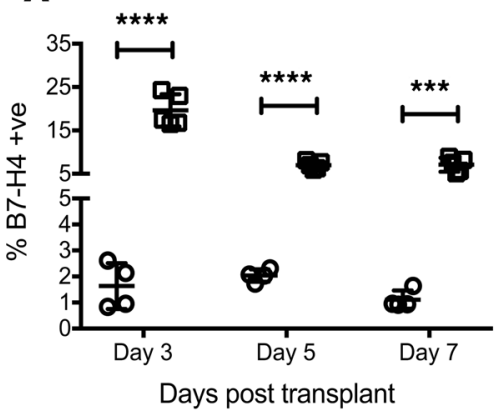

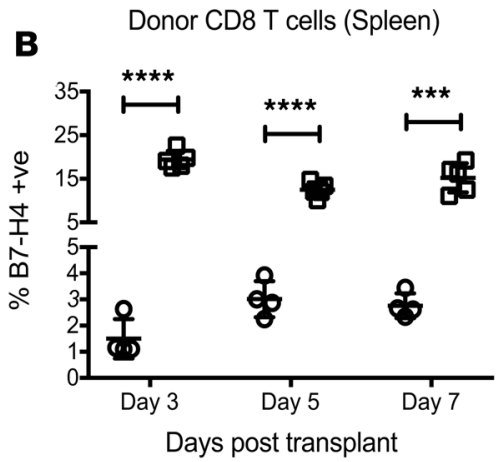

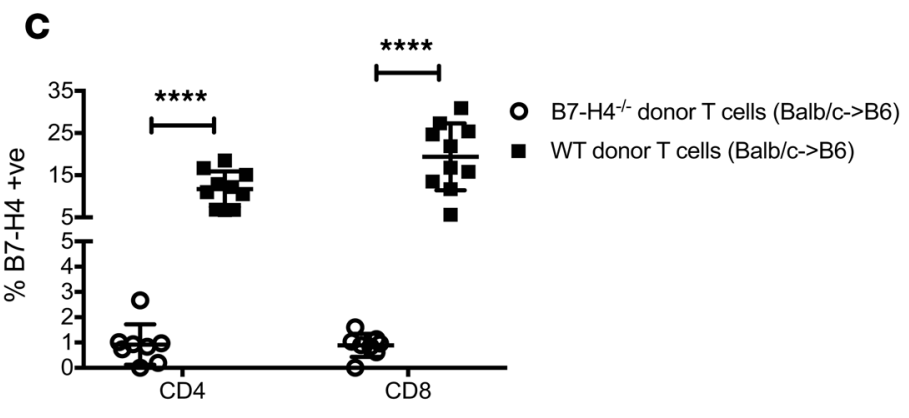

Day 5 post transplant

D

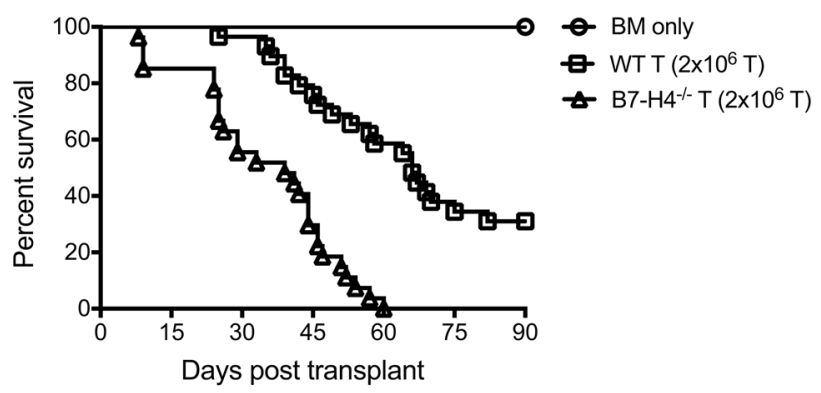

$\mathbf{F}$

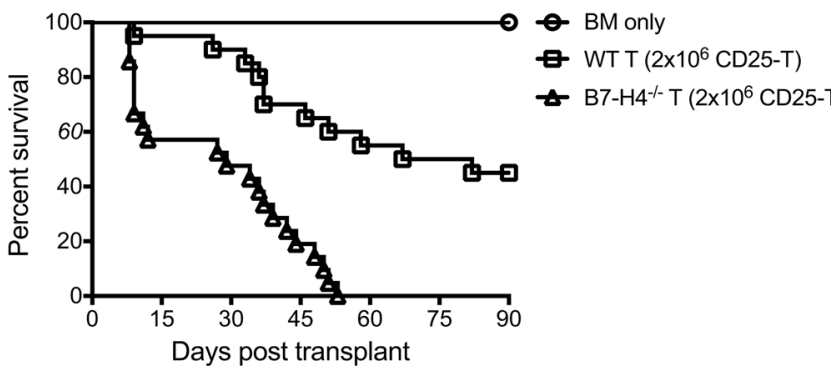

$\mathbf{E}$

Balb/c->B6

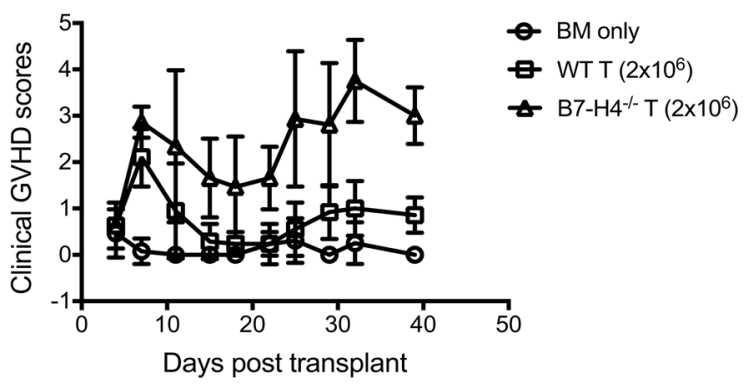

G

$\mathrm{Balb} / \mathrm{c}->\mathrm{B} 6$

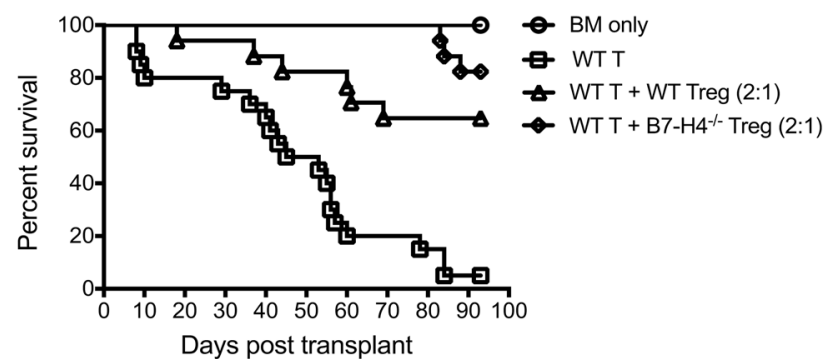

Figure 5. B7-H4 ${ }^{-/-}$donor T cells cause increased GVHD lethality that is independent of donor Treg function. (A and B) Lethally irradiated B6 or BALB/C recipients were infused with $10^{7}$ WT B6 BM cells plus $2 \times 10^{6}$ B6 Ly5.2 (CD45.1+) T cells. Mice were sacrificed ( $n=4-5 / g r o u p /$ day) on day 3, day 5, and day 7 after BMT, and splenic donor $\mathrm{CD}^{+}(\mathbf{A})$ and $\mathrm{CD}^{+}$(B) T cells were analyzed by flow cytometry for B7-H4 expression. Data are representative of 2 independent experiments. (C) Lethally irradiated B6 recipients were infused with $10^{7}$ WT BALB/C BM cells plus $2 \times 10^{6} \mathrm{WT} B A L B / C$ or B7-H4 $4^{-/-}$T cells. Mice were sacrificed on day 5 after $\mathrm{BMT}$, and splenic donor $\mathrm{CD} 4^{+}$and $\mathrm{CD} 8^{+} \mathrm{T}$ cells were analyzed by flow cytometry for $\mathrm{B} 7-\mathrm{H} 4$ expression. Pooled data from 2 independent experiments ( $n=8-10 /$ group). ( $\mathbf{D}$ and $\mathbf{E}$ ) Lethally irradiated B6 recipients were infused with $10^{7}$ WT BALB/c BM cells alone or with $2 \times 10^{6}$ WT BALB/c or B7-H4 $4^{-/-}$T cells. (D) Kaplan-Meier survival plot represents pooled data from 3 independent experiments ( $n=21-29 / g r o u p ; B M+T$ cells: recipients of WT versus B7-H4 ${ }^{-/-}$donor $T$ cells, $P<0.0001$ ). (E) Transplanted mice were evaluated for clinical GVHD. BM + T cells: recipients of WT versus B7-H4 ${ }^{-1-}$ donor $\mathrm{T}$ cells, $P<0.0001$ on d7, d15, $\mathrm{d} 18, \mathrm{~d} 22, \mathrm{~d} 25, \mathrm{~d} 29, \mathrm{~d} 32$, and d39; $P=0.0043$ on d11. Pooled data from 2 independent experiments $(n=13-19 / \mathrm{group})$. (F) Lethally irradiated B6 recipients were infused with $10^{7}$ WT BALB/c BM cells alone or with $2 \times 10^{6}$ WT BALB/C or B7-H4-/- CD25-depleted T cells. Kaplan-Meier survival plot represents pooled data from 2 independent experiments ( $n=14-21 /$ group; BM + T cells: recipients of WT versus B7-H4 $4^{-1-}$ donor T cells, $\left.P<0.0001\right)$. (C) Lethally irradiated B6 recipients were infused with $10^{7}$ WT BALB/c BM cells alone, or with $2.5 \times 10^{6}$ WT BALB/c T cells, or with $2.5 \times 10^{6}$ WT BALB/c T cells plus $1.25 \times 10^{6}$ WT BALB/c Tregs or $1.25 \times 10^{6} \mathrm{~B} 7-\mathrm{H}^{-1-}$ Tregs. Kaplan-Meier survival plot represents pooled data from 2 independent,experiments $(n=13-20 /$ group; WT T cells versus WT T cells + WT Tregs, $P=0.0001$; WT T cells versus WT T cells + B7- H4 $4^{-1-}$ Tregs, $P<0.0001$; WT T cells + WT Tregs versus WT T cells $+\mathrm{B} 7-\mathrm{H} 4^{-1-}$ Tregs, $\left.P=0.1732\right) .($ A-C and E) Data represent mean $\pm \mathrm{SEM}$. $P$ values were calculated by 2 -tailed $t$ test $(\mathbf{A}-\mathbf{C}$ and $\mathbf{E})$ or log-rank test $(\mathbf{D}$, $\mathbf{F}$, and $\mathbf{G})$. 

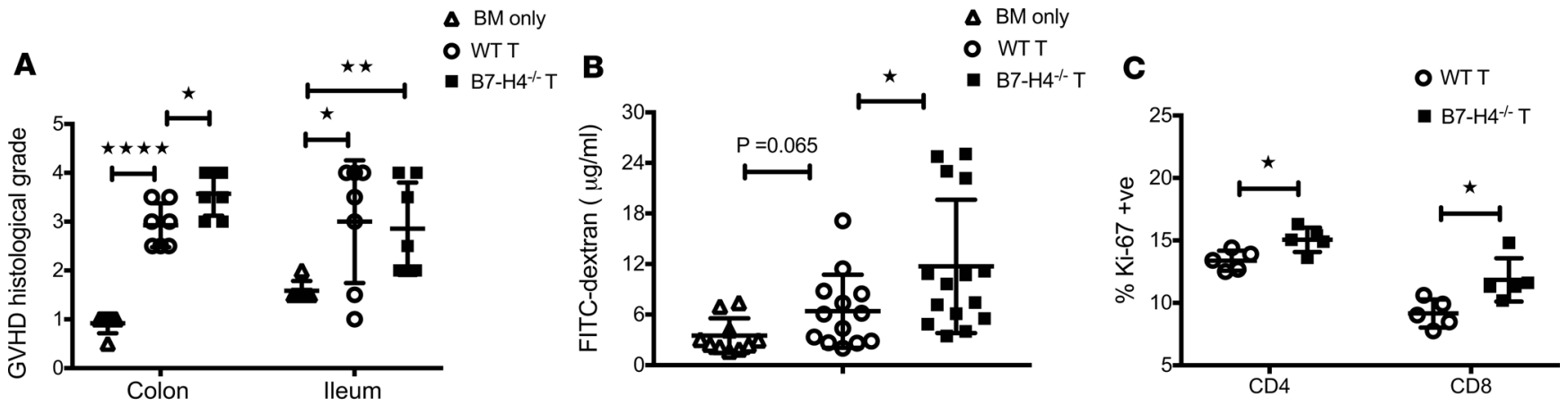

D

E
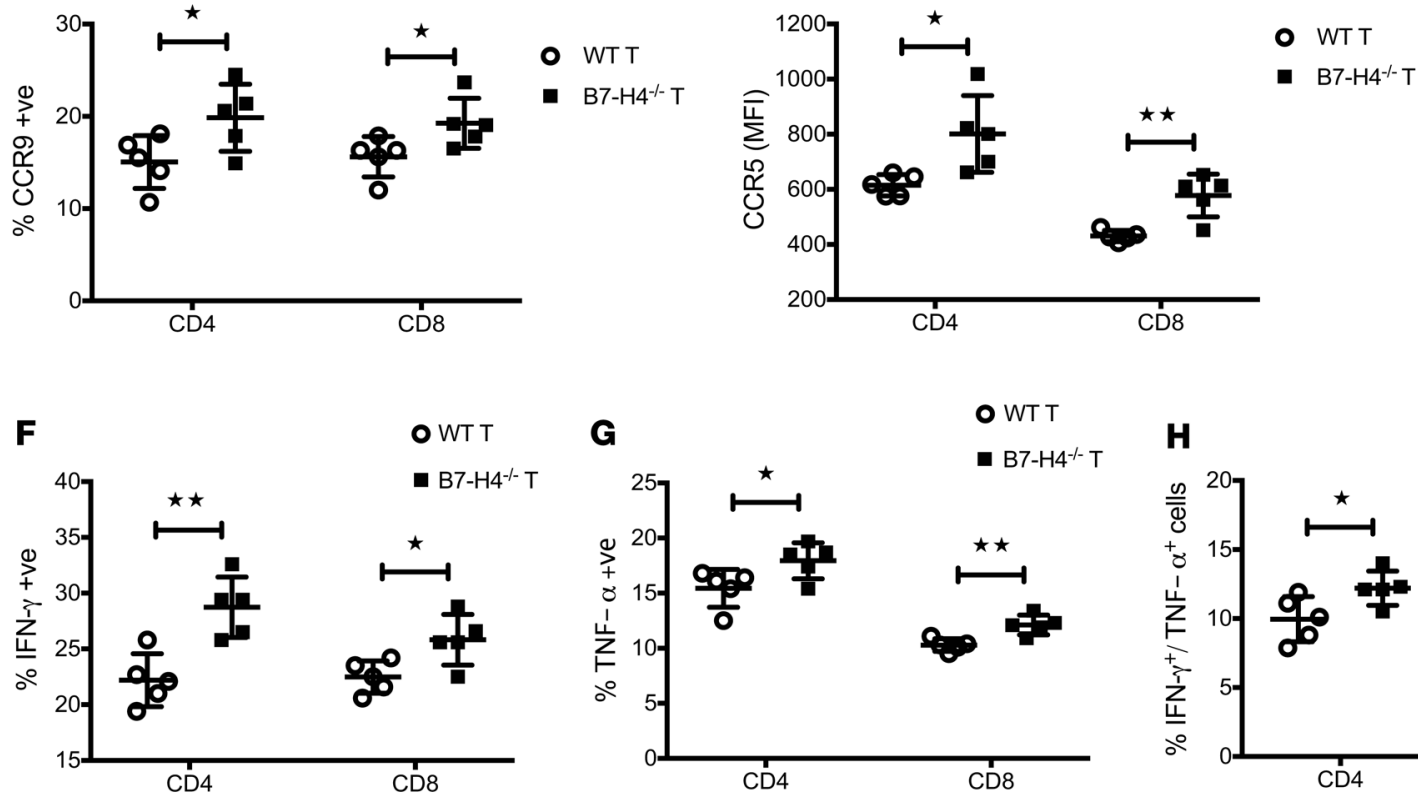

G

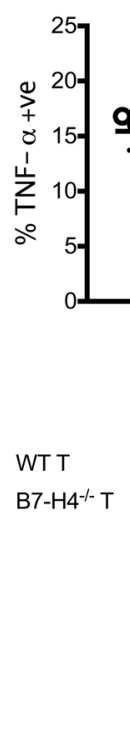

○ WT T

- $\mathrm{B} 7-\mathrm{H} 4^{-1-} \mathrm{T}$

\section{H}

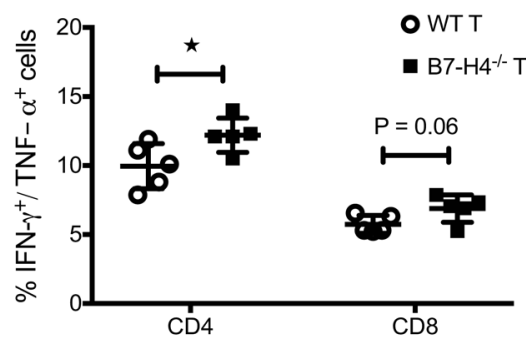

J

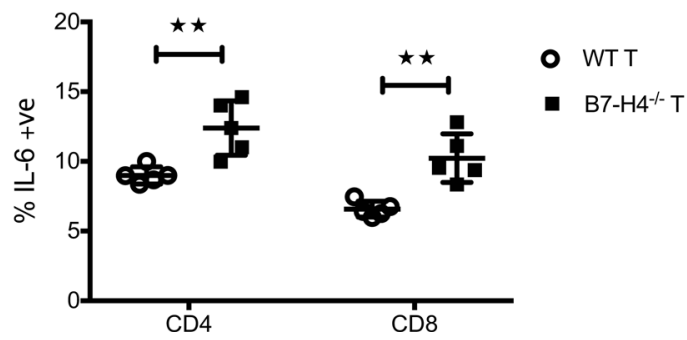

Figure 6. Elevated T effector function by B7-H4-- donor T cells promotes increased gut injury in recipients. Lethally irradiated $\mathrm{B} 6$ recipients were infused with $10^{7}$ WT BALB/c BM cells alone or with $2 \times 10^{6} \mathrm{WT}$ BALB/c or B7-H4 $4^{-/-}$purified T cells. (A) Mice were sacrificed on day 30 after BMT ( $n=6-7 /$ group), and H\&E-stained tissue sections were scored for CVHD. Data are representative of 2 independent experiments. (B) FITC-dextran was administered orally on day 30 after BMT and plasma levels were measured after 4 hours. Pooled data obtained from 2 independent experiments ( $n=10-15$ mice/group). (C-J) Mice were sacrificed on day 29 after BMT ( $n=10$ /group), and lymphocytes isolated from colon ( 2 colons were pooled to make 1 pooled sample and 5 pooled samples per group) were analyzed by flow cytometry. Donor CD4 ${ }^{+}$and CD8 ${ }^{+} T$ cells were analyzed for surface expression of CCR9 (D) or CCR5 (E). Donor T cells were also analyzed for intracellular expression of Ki-67 (C), IFN- $\gamma$ (F), TNF- $\alpha$ (G), IFN- $\gamma /$ TNF- $\alpha$ (H), CD107a/ IFN- $\gamma$ (I), or IL-6 (J). (C-J) Data are representative of 2 independent experiments. Data represent mean \pm SEM. $P$ values were calculated by 2 -tailed $t$ test. ${ }^{*} P<0.05 ;{ }^{* *} P<0.01 ;{ }^{* *} P<0.001 ;{ }^{* * *} P<0.0001$.

In studies designed to assess GVL responses with $\mathrm{B} 7-\mathrm{H} 4^{-/-}$versus WT donor T cells, lethally irradiated B6 recipients were infused with WT BALB/c TCD BM plus 2 times the lethal dose of B6 recipient strain TBL-12 $2^{\text {luc }}$-lymphoma cells on day 0 . Cohorts were administered with WT BALB/c or B7-H4 ${ }^{-1-}$ T cells at a dose of $3 \times 10^{6}$ (Figure 8, A, B and E) on day 0 . Mice that received TCD BM and TBL-12 $2^{\text {luc }}$ cells alone died within day 20 after BMT as a result of tumor burden (Figure 8, A, B, and E), whereas recipients with WT 
A

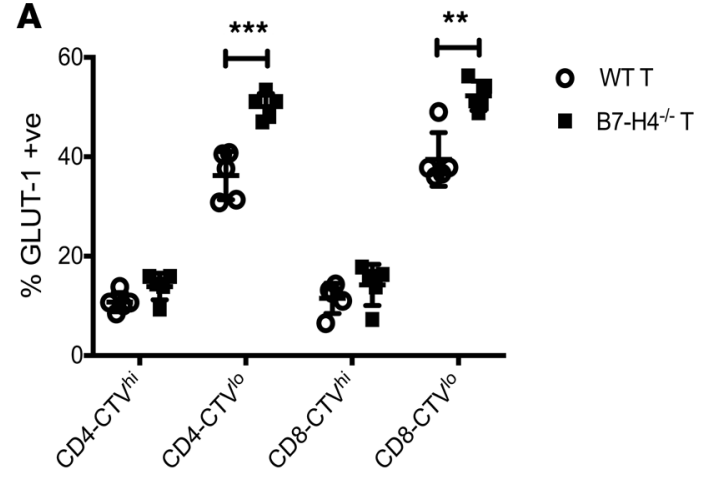

C

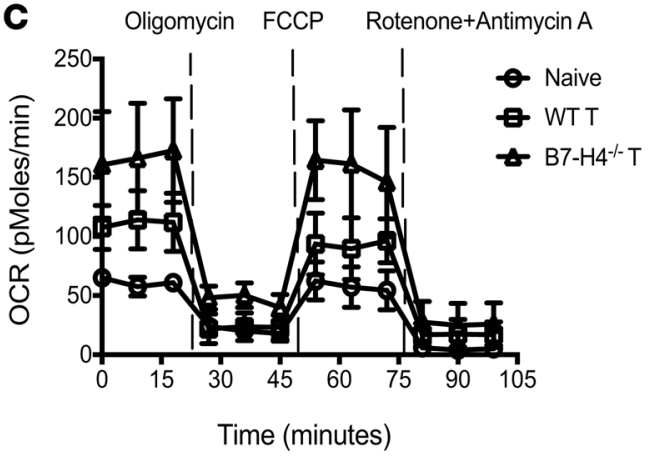

B

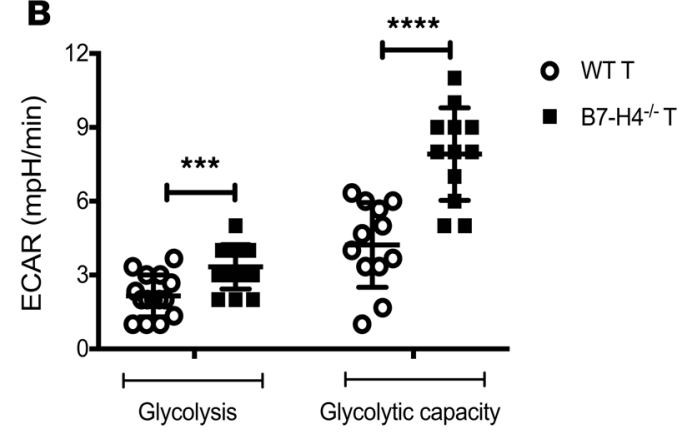

D

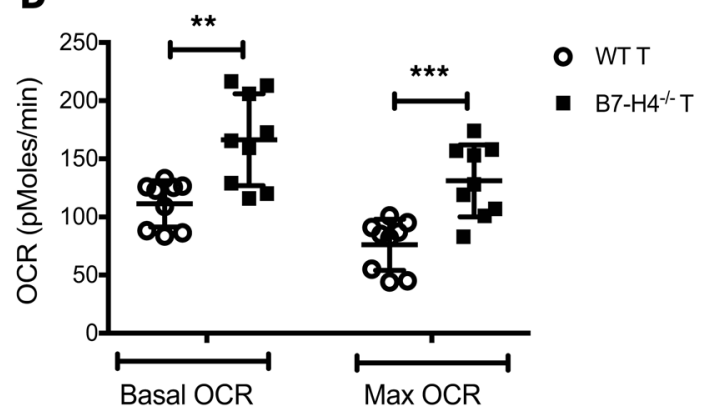

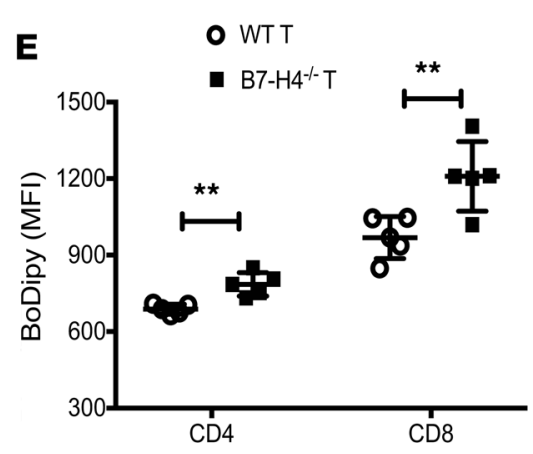

$\mathbf{F}$

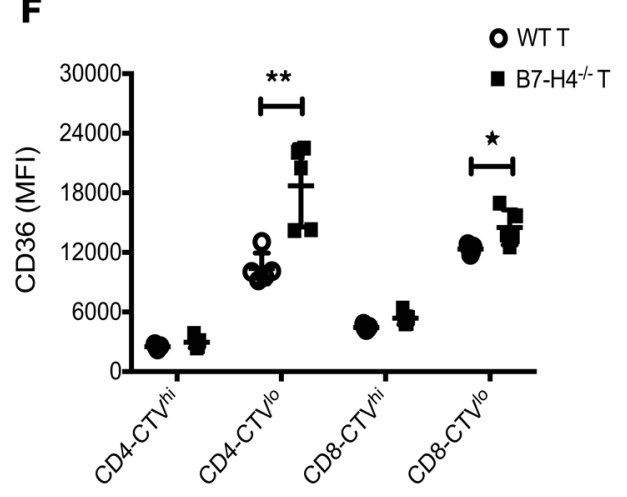

- WT T

G

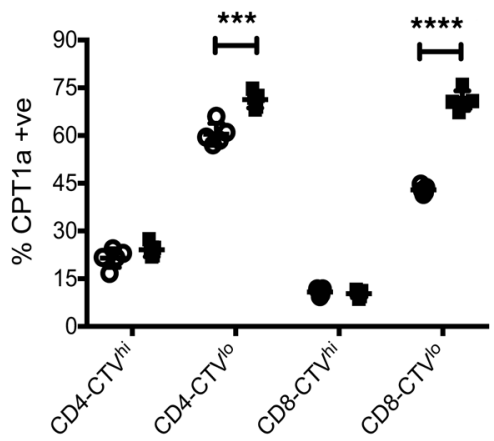

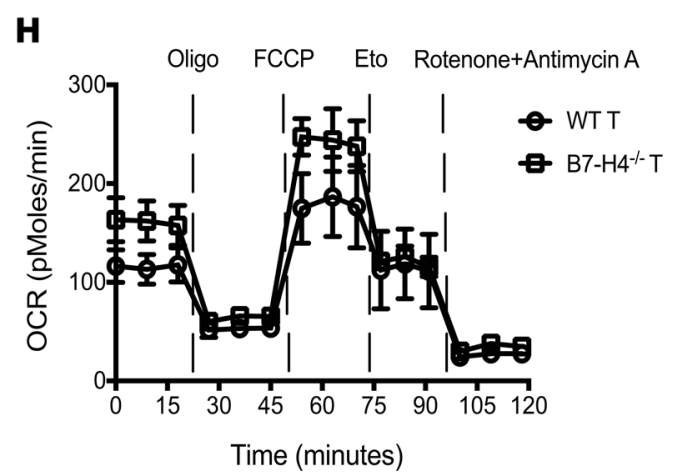

I

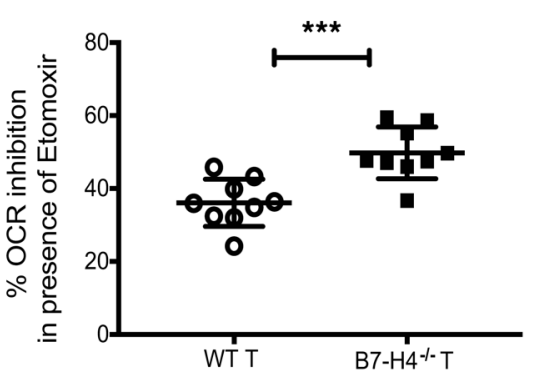

Figure 7. Metabolic alterations in B7-H4-/- versus WT donor T cells during CVHD. Lethally irradiated B6 recipients were infused with $10^{7}$ WT BALB/C BM cells plus $6 \times 10^{6} \mathrm{CTV}$-labeled or unlabeled WT BALB/C or B7- $\mathrm{H}^{-/-}$purified T cells. Mice were sacrificed on day 5 after BMT, and experiments were performed as described. (A) Splenocytes were analyzed by flow cytometry for intracellular expression of GLUT1 in undivided (CTVhi) and divided (CTV ${ }^{10}$ ) donor T cells. (B) Extra-cellular acidification rate (ECAR) of purified donor T cells was measured after addition of glucose, oligomycin, and 2-deoxyglucose (2-DG). Basal glycolysis was measured after addition of glucose, and glycolytic capacity was measured after addition of oligomycin. The graph represents pooled data from 5 time points to measure glycolysis and pooled data from 4 time points to measure glycolytic capacity. (C) Oxygen consumption rate (OCR) of purified donor T cells was measured after addition of oligomycin, FCCP, and rotenone plus antimycin A. T cells from naive WT BALB/c mice ( $n=$ 4) were included as control. (D) Basal OCR (resting OCR - rotenone plus antimycin A OCR) was measured before addition of oligomycin, and maximal OCR 
was measured after addition of FCCP subtracting nonmitochondrial OCR (rotenone plus antimycin A OCR). The graph represents pooled data from 3 time points to measure basal OCR or maximal OCR. Splenic donor T cells were analyzed by flow cytometry for BoDipy (E) or CD36 (F) expression. (G) Splenocytes were also analyzed by flow cytometry for intracellular expression of CPT1a in undivided and divided donor T cells. (H and I) OCR of purified donor T cells was measured after addition of oligomycin, FCCP, etomoxir (Eto), and rotenone plus antimycin A. (I) Percent inhibition was calculated using pooled data from 3 time points after addition of etomoxir. (A and $\mathbf{E}-\mathbf{G}$ ) Data are representative of 5 mice per group from 2-3 independent experiments. (B-D, $\mathbf{H}$, and I) Data are representative of 12 mice per group from $2-3$ independent experiments. MFI, mean fluorescence intensity. Data represent mean \pm SEM, and $P$ values were calculated by 2 -tailed $t$ test. ${ }^{*} P<0.05$; ${ }^{* *} P<0.01$; ${ }^{* *} P<0.001 ;{ }^{* * *} P<0.0001$.

or B7-H4 ${ }^{-/-}$donor $\mathrm{T}$ cells had no tumor growth (Figure 8, A and E) and died as a result of GVHD (Figure $8 \mathrm{~B})$. GVHD was accelerated in recipients of $\mathrm{B} 7-\mathrm{H} 4^{-/-}$versus WT donor T cells $(P=0.0284)$. A lower dose of donor T cells $\left(2 \times 10^{6}\right)$ replicated the findings in mice that received TBL-12 $2^{\text {luc }}$ cells with WT or B7-H4 ${ }^{-1-}$ donor T cells in which mice were tumor-free (Figure 8, C and F) but died as a result of GVHD (Figure 8D).

Lastly, to reduce the alloresponse further in an effort to uncover a difference in GVL, we performed GVHD studies in the absence of tumor cells, using an even lower WT or B7-H4 ${ }^{-/}$donor T cell dose $(1 \times$ $\left.10^{6}\right)$. As shown in Supplemental Figure $8, \mathrm{~K}$ and $\mathrm{L}$, the higher T cell dose $\left(3 \times 10^{6}\right)$ accelerated GVHD lethality as expected $\left(P=0.04\right.$, recipients of WT versus $\mathrm{B} 7-\mathrm{H} 4^{-/-}$donor T cells). The median survival times for higher and lower WT T cell doses in this experiment were 45 days and 72 days, respectively, and there was no difference in GVHD lethality at the lower $\mathrm{T}$ cell dose. These findings are consistent with our hypothesis that aggressive GVHD lethality is associated with B7-H4 upregulation on donor T cells (Figure 5, A-C, and Supplemental Figure 8, A and B), whereas less aggressive GVHD is insufficient to cause B7-H4 upregulation to levels impacting GVHD lethality. As such, additional tumor studies were not pursued. Collectively, these data suggest that B7-H4 expression on donor T cells is regulated by the severity of GVHD and further suggest that GVHD and GVL responses are linked under these conditions.

\section{Discussion}

Here we have shown that within the first week after BMT, B7-H4 expression was increased in host cells within GVHD organs and hematopoietic cells, and to a lesser extent parenchymal cells B7-H4 expression was required to inhibit acute lethality. B7-H4 cell surface expression on donor T cells was also elevated by day 5 after BMT and its absence lead to accelerated GVHD. In both B7-H4 ${ }^{-/-}$recipients and recipients of $\mathrm{B}_{\mathrm{H}} 4^{-/-}$donor $\mathrm{T}$ cells, augmented GVHD lethality was associated with increased donor $\mathrm{T}$ cell activation, proliferation, expansion, gut homing receptor and proinflammatory cytokine expression, along with reduced coinhibitory pathway expression and reduced expression of apoptotic proteins. However, enhanced substrate transporter expression on donor $\mathrm{T}$ cells fueled multiple metabolic pathways that were engaged to provide energy to highly proliferative $\mathrm{B} 7-\mathrm{H} 4^{-/-}$versus WT donor T cells. Because B7H4 ${ }^{-/}$Tregs were at least as suppressive as WT Tregs in vivo, and Treg-depleted T cells from B7-H4 ${ }^{-/}$versus WT donors caused heightened lethality, T effector cell expression of B7-H4 is critical in regulating acute GVHD.

In contrast to other B7-family members, B7-H4 mRNA is widely expressed $(3,4)$. Despite the widespread expression of B7-H4 mRNA, the presence of B7-H4 protein on the surface of normal cells is limited (4). B7-H4 expression on hematopoietic cells can be induced after in vitro stimulation (4) and the expression of B7-H4 changes in various strains of mice with age (20). B7-H4 cell surface expression appears to be activation-induced, as noted in donor T cells during GVHD in our studies.

Our studies in which host B7-H4 deficiency results in accelerated GVHD lethality support the immunosuppressive function of B7-H4 in different disease models (7, 10-16). Strong similarities to other B7 homologs with negative regulatory function exist. For example, we reported that PD-1 ligands in host parenchymal and hematopoietic cells are upregulated during acute GVHD and that PD-L1 $1^{-/}$recipients or WT recipients given blocking anti-PD-L1 mAb resulted in accelerated acute GVHD lethality (30). Whereas PD-L1 expression in host parenchymal cells is essential in downregulating acute GVHD, host B7-H4 expression in hematopoietic cells dominated over parenchymal cells in suppressing lethality. In addition, the B7 family member B7-H3 also is upregulated in GVHD target organs and B7-H3 ${ }^{-/}$versus WT recipients are hypersusceptible to acute GVHD lethality (39). In aggregate with our studies using B7-H4 ${ }^{-/-}$recipient mice, host deficiency of each of several B7 family members with coinhibitory function significantly accelerated GVHD lethality compared with WT recipients. Taken together, B7 family members with coinhibitory function appear to be upregulated in GVHD tissues and donor T cells during the inflammatory process of GVHD, likely representing a means by which immune homeostasis can be induced within the 
A

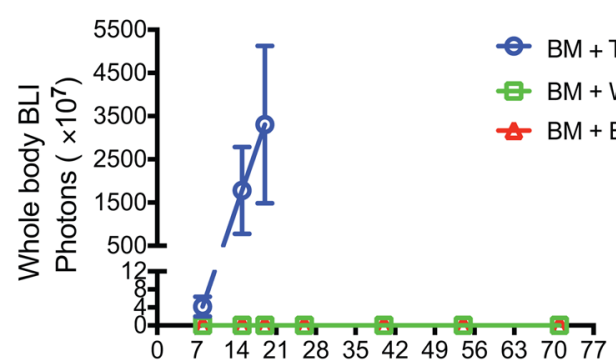

Days post transplant

C

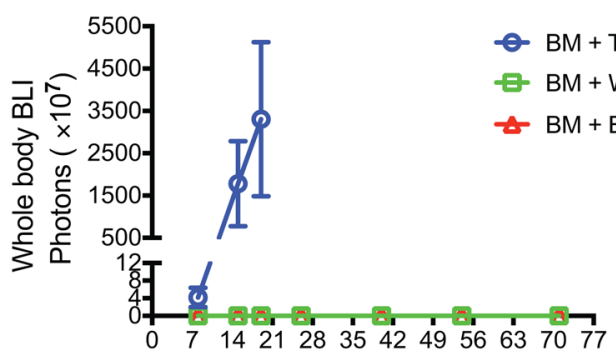

Days post transplant
B

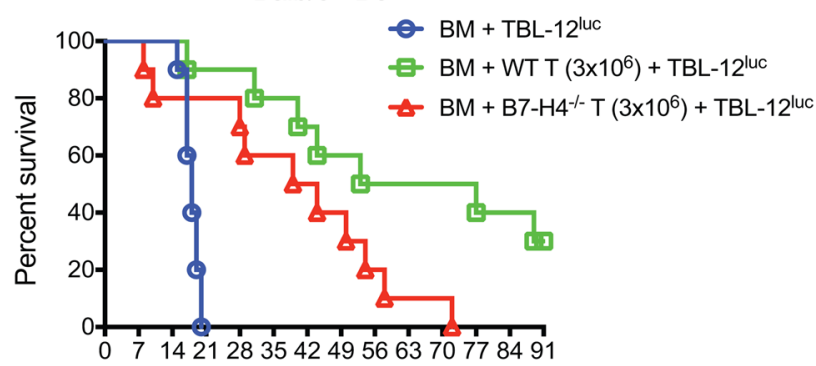

Days post transplant

D Balb/c->B6

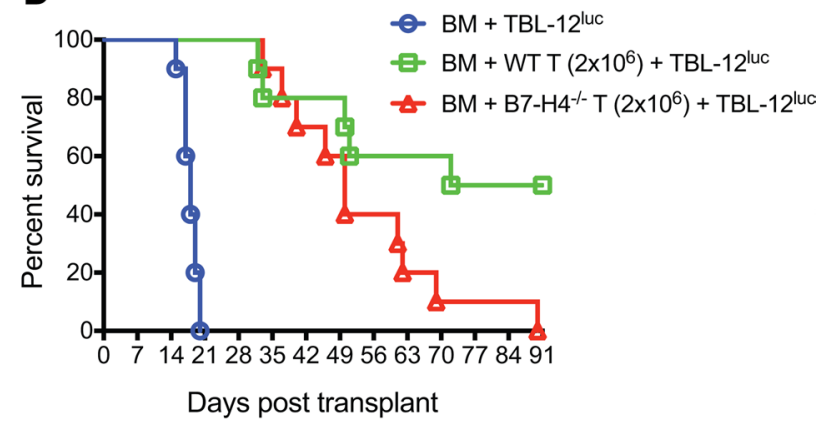

E

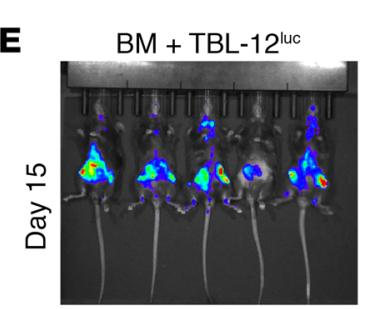

$\mathrm{BM}+\mathrm{WT} \mathrm{T}\left(3 \times 10^{6}\right)$ + TBL-12luc
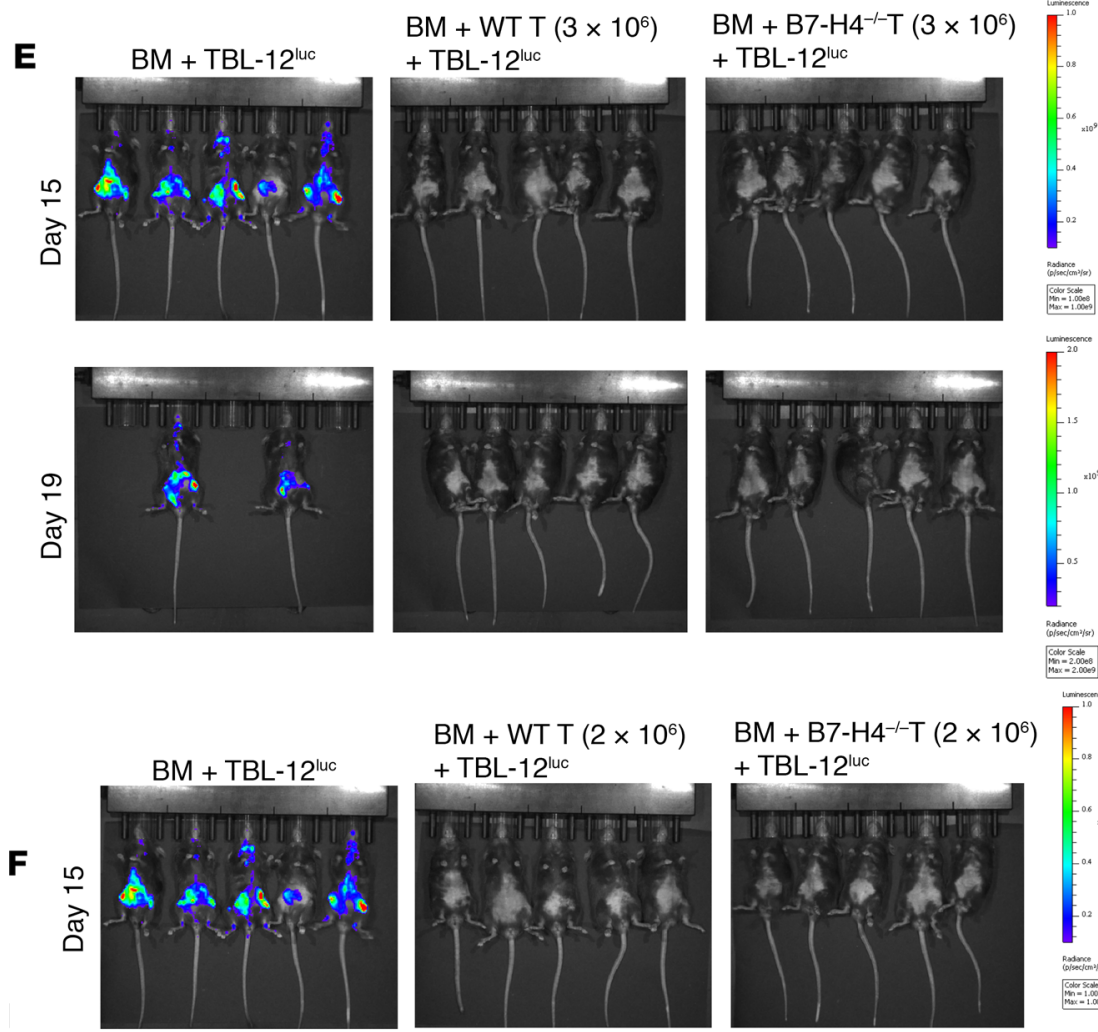
+ TBL-12 luc

$\mathrm{BM}+\mathrm{B} 7-\mathrm{H} 4^{-l-\mathrm{T}}\left(2 \times 10^{6}\right)$ + TBL $-12^{\text {luc }}$

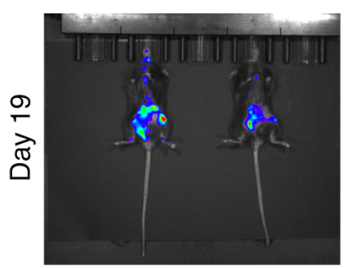

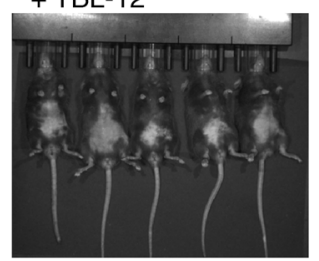
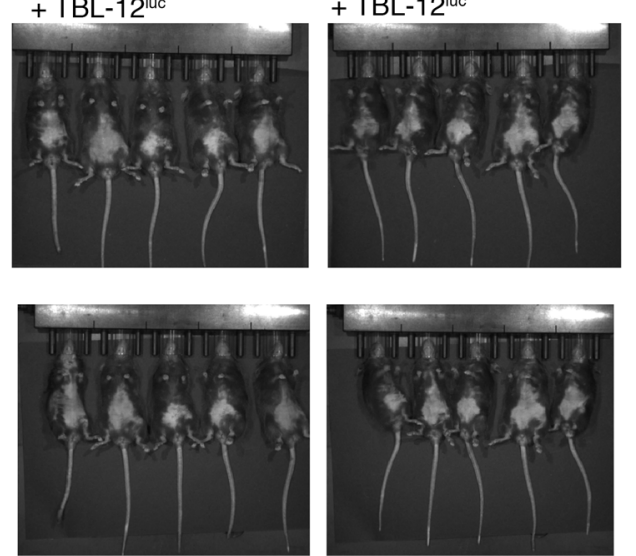

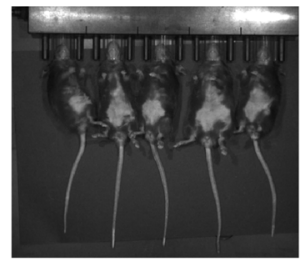

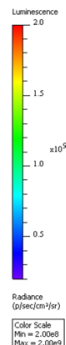

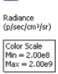
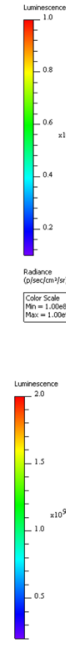

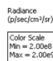

Figure 8. GVL effect is retained in recipients of WT versus B7-H4 ${ }^{-/-}$donor T cells. (A, B, and E) Lethally irradiated $B 6$ recipients were infused with $10^{7} \mathrm{~T}$ cell-depleted WT BALB/c BM cells plus $5 \times 10^{3}$ TBL-12'uc-lymphoma cells or lethally irradiated $B 6$ recipients were infused with $10^{7} \mathrm{~T}$ cell-depleted WT BALB/c BM cells plus $5 \times 10^{3}$ TBL-12 /uc_lymphoma cells along with $3 \times 10^{6} \mathrm{WT}$ $\mathrm{BALB} / \mathrm{c}$ or B7- $\mathrm{H} 4^{-/-}$purified T cells on day $\mathrm{O}(n=10$ mice/group). (C, D, and F) Lethally irradiated B6 recipients were infused with $10^{7} \mathrm{~T}$ cell-depleted WT BALB/c BM cells plus $5 \times 10^{3} \mathrm{TBL}-12^{\text {luc }}$ lymphoma cells or lethally irradiated $B 6$ recipients were infused with $10^{7} \mathrm{~T}$ cell-depleted WT BALB/C BM cells plus $5 \times 10^{3} \mathrm{TBL}-12^{l u c}-$ lymphoma cells along with $2 \times 10^{6}$ WT BALB/C or B7-H4 $4^{-/-}$purified T cells on day 0 ( $n=10$ mice/group). ( $A$ and $\mathbf{C}$ ) Tumor growth was monitored by LUC imaging on day 8 , day 15 , day 19 , day 26 , day 40 , day 54 , and day 71 after BMT. BLI, bioluminescence imaging. (B) Kaplan-Meier survival plot of transplanted mice is shown (recipients of WT versus B7- $\mathrm{H}^{-}$ 1- donor T cells, $P=0.0284$ ). (D) Kaplan-Meier survival plot of transplanted mice is shown (recipients of WT versus B7-H4 $4^{-1-}$ donor T cells, $P=0.0258$ ). ( $E$ and $\mathbf{F}$ ) In vivo BLI of TBL-12 $2^{l u c_{-}}$ lymphoma cells on day 15 and day 19 after BMT. The scale to the right of the images describes the color map for the photon count. (B and $\mathbf{D}) P$ values were calculated by log-rank test. (A-F) Data were obtained from 1 experiment and same control transplanted mice (BM + TBL-12 ${ }^{l u c}$ ) were used in $\mathbf{A}$, $\mathbf{B}$, and $\mathbf{E}$; and in $\mathbf{C}, \mathbf{D}$, and $\mathbf{F}$. 
sites of inflammation (40). Thus, the degree of the inflammatory response that can be influenced by both the conditioning regimen intensity and the aggressiveness of GVHD-induced lethality may increase B7-H4 upregulation. This hypothesis is consistent with the finding that $\mathrm{B} 7-\mathrm{H} 4$ upregulation on host hematopoietic and parenchymal cells is seen in allogeneic but not syngeneic donor T cell recipients on day 5 after BMT. In an analogous fashion to other B7 family members with coinhibitory function, such as PD-1 (41) and B7-H3 (39), T cells from B7-H4 ${ }^{-/-}$mice caused hyperacute GVHD lethality. Thus, the lack of B7-H4 expression on donor T cells leading to accelerated GVHD lethality parallels our findings with B7-H3, indicating that elimination of a negative regulator can increase allogeneic donor anti-host $\mathrm{T}$ cell responses. We have previously reported that B7-1 expression on donor T cells can be inhibited by CTLA-4 expression on donor T cells in a T-T interaction (42). In an analogous fashion, upregulated B7-H4 on donor T cells may bind B7-H4 ligand on donor T cells or host cells. Although Tregs can modulate GVHD severity and lethality $(22,34)$ and a link between B7-H4Ig and Tregs has been reported in an experimental autoimmune encephalomyelitis model (14), GVHD lethality in B7-H4 ${ }^{-/-}$versus WT recipients was similarly accelerated by the infusion of Treg-replete or Treg-depleted donor WT T cells, similar to accelerated lethality seen in WT recipients given donor Treg-replete or Treg-depleted B7-H4 ${ }^{-/-}$versus WT T cells. Although blocking anti-PD-1 mAb phenocopied the findings with PD-1/-- donor T cells, neither of 2 known blocking anti-B7-H4/B7S1/B7x mAbs (clone 19D6, ref. 7; or clone 1H3, ref. 43) given at their optimal doses and schedules accelerated GVHD-induced lethality (Supplemental Figure 1E and data not shown), similar to blocking anti-B7-H3 mAbs that failed to phenocopy B7-H3 ${ }^{-/}$recipients or donor T cells in the context of GVHD (39). Although B7-H4Ig versus irrelevant fusion protein administration could attenuate GVHD lethality in B10.BR recipients of WT B6 BM and splenocytes $\left(15 \times 10^{6}\right)(P=0.017)$, the effect was modest with only a 4-day prolongation of MST and overall survival (data not shown). These data highlight the main limitation of our study, kinetics, site, functional properties of and availability of mAbs against the as-yet unknown B7-H4 receptor and point to the need for future studies testing other mAbs directed to B7-H4 or its receptor.

A likely contributory mechanism for accelerated GVHD lethality in $\mathrm{B} 7-\mathrm{H} 4^{-/-}$recipients or recipients of $\mathrm{B} 7-\mathrm{H}_{4}^{-/-}$donor $\mathrm{T}$ cells is increased donor $\mathrm{T}$ cell infiltration in GVHD target tissues. Our findings of higher CD127 and lower annexin V and FasL expression provide a downstream mechanism to explain the higher proliferation and absolute numbers of donor T cells. IL-7 signaling via CD127 promotes the upregulation of antiapoptotic protein Bcl-xL along with Bcl-2 in $\mathrm{CD}^{+}$effector/memory $\mathrm{T}$ cells (44). In addition, CD127 signaling is necessary but not sufficient for the formation of memory $\mathrm{CD}^{+} \mathrm{T}$ cells during viral infection (45) and for the transition of $\mathrm{CD} 4^{+} \mathrm{T}$ effector into memory cells (46). Higher $\mathrm{T}$ cell gut homing receptor and proinflammatory cytokine (IFN- $\gamma, \mathrm{TNF}-\alpha, \mathrm{IL}-6)$ expression may account for, in part, to the greater gut injury seen in $\mathrm{B} 7-\mathrm{H} 4^{-/}$versus WT recipients (47). IFN- $\gamma$ may upregulate host $\mathrm{MHC}$, costimulatory molecules, or it may stimulate production of other inflammatory cytokines (48) that can function as a GVHD effector mechanism. These data are consistent with our GVHD studies in which PD-1 pathway blockade results in high IFN- $\gamma$ production (41) and findings by other clinicians suggesting that IFN- $\gamma$ can cause tissue damage in the gut and liver during acute GVHD (49).

During GVHD, the metabolic demand of donor $\mathrm{T}$ cells in response to activation leads to metabolic adaptations to facilitate expansion and differentiation. These include induction of aerobic glycolysis, OXPHOS, glutaminolysis, increase in synthesis of biomolecules, lipid synthesis or oxidation, and amino acid uptake (31, 50-54). We (30), and others (29) have shown that GVHD results in high metabolic demands for $\mathrm{T}$ effectors, increasing their dependency on aerobic glycolysis. Glucose uptake provides a key metabolic control point through the GLUT family of glucose transporters. GLUT1 can promote T effectors because GLUT1 over-expression selectively increases T effector frequency and leads to inflammatory disease (55), whereas GLUT1 deficiency decreases T effector expansion and the ability to induce inflammation (56). GLUT1 expression was increased in proliferating WT donor T cells in B7-H4 ${ }^{-/}$recipients and $\mathrm{B} 7-\mathrm{H}^{-/-}$donor $\mathrm{T}$ cells in WT recipients along with increased glycolysis and OXPHOS, suggesting that proliferating alloreactive T cells use both aerobic glycolysis and OXPHOS for ATP synthesis.

Mitochondrial electron transport chain is a major source of ROS in mammalian cells in the form of superoxide (57). Mitochondrial ROS production also increases in proportion with $\Delta \Psi \mathrm{m}$ because hyperpolarization of $\Delta \Psi \mathrm{m}$ can prolong the half-life of reactive intermediates, which increases the formation of superoxide (58). Therefore, it is not surprising that increased OXPHOS by proliferative alloreactive $\mathrm{T}$ cells would exhibit increased ROS production. Although memory T cells preferentially use FA metabolism (59), T effectors can also rely on FAO during GVHD (31). Increased FA uptake and FAO and enhanced 
susceptibility to pharmacologic inhibition of FAO by etomoxir in donor T cells suggest that B7-H4 acts as a negative regulator of $\mathrm{T}$ cell activation, and loss of $\mathrm{B} 7-\mathrm{H} 4$ expression may provide a cell intrinsic positive stimulus for FA metabolism in alloreactive T cells undergoing clonal expansion.

Taken together, our results demonstrate the important function of B7-H4 in regulating acute GVHD. Future studies will further warrant B7-H4 expressing host cells responsible for GVHD attenuation. B7-H4 expression was upregulated in GVHD target organs and on donor T cells early after BMT. The rapid onset of mortality in $\mathrm{B} 7-\mathrm{H}_{4}^{-/-}$recipients or in $\mathrm{WT}$ recipients of $\mathrm{B} 7-\mathrm{H}_{4}{ }^{-/}$donor $\mathrm{T}$ cells was associated with increased proliferation, activation, and Th1 cytokine production, along with increased homing in GVHD target tissues including gut, as well as loss of intestinal epithelial integrity. Greater understanding of the function of B7-H4 expression by activated donor T cells will potentially provide new insight into the regulation of GVHD and suggest clinically useful strategies to selectively increase B7-H4 expression on donor T cells, or that increasing B7-H4 expression early after BMT may be clinically useful to prevent GVHD.

\section{Methods}

Experimental animals. Female BALB/c $\left(\mathrm{H}-2^{\mathrm{d}}\right)$ mice were purchased from Charles River Laboratories. Female C57BL/6 (H-2 $2^{\mathrm{b}}$; termed B6), B10.BR $\left(\mathrm{H}-2^{\mathrm{k}}\right)$, and congenic BALB/c mice $\left(\mathrm{CD} 45.1^{+}\right)$were purchased from The Jackson Laboratory. Ly5.2 (H-2 $)$ congenic mice (CD45. $\left.1^{+}\right)$were purchased from Charles River Laboratories. B7-H4 ${ }^{-/-}$mice in BALB/c background (provided by James P. Allison at the University of Texas, Houston, Texas, USA) were backcrossed for 4 additional generations at the University of Minnesota before use in experiments. The luciferase-expressing transgenic B6-L2G85 (H-2 , Thy1.1) mice were obtained from Robert S. Negrin (Stanford University, Stanford, California, USA). Mice were bred and housed in a specific pathogen-free facility at the University of Minnesota and used at 8-14 weeks of age

Bone marrow transplantation. BM transplantations were performed as described previously (30). Briefly, recipient mice received total body irradiation at a lethal dose (BALB/c 700 cGy; B10.BR 1000 cGy; B6 1100 cGy) by X-ray on day -1 . BM cells alone or with purified T cells (purity >97\%), CD25-depleted T cells, $\mathrm{T}$ cells plus Tregs, or splenocytes were infused i.v. on day 0 . Mice were evaluated for evidence of clinical GVHD as described previously (60).

Quantitative PCR (qPCR). RNA was purified using RNeasy Plus Mini Kit (QIAGEN) and cDNA was synthesized from total RNA using the SuperScript VILO cDNA Synthesis Kit (Thermo Fisher Scientific). For qRT-PCR experiments, reactions containing the TaqMan Gene Expression Master Mix (Thermo Fisher Scientific) and probes for B7-H4 (Mm00628552_m1), or glyceraldehyde-3-phosphate dehydrogenase (Mm99999915_g1) as the endogenous control gene were run on QuantStudio 3 Real-Time PCR System (Thermo Fisher Scientific) and analyzed with QuantStudio Design and Analysis Software v1.4 (Applied Biosystems). The $2^{-\Delta \Delta C_{t}}$ method was used to generate RQ for quantifying B7-H4 expression in samples in comparison with unmanipulated WT mice.

BMchimeras. WT BALB/c or B7-H4 ${ }^{-/-}$mice were lethally irradiated and reconstituted with BM cells from $\mathrm{B} 7-\mathrm{H}_{4}{ }^{--}$or WT BALB/c mice, respectively. To create control chimeras, WT BALB/c recipients were lethally irradiated and reconstituted with BALB/c CD45.1 $1^{+} \mathrm{BM}$ cells. After 3 months, chimerism was confirmed by evaluating CD45.1 expression on CD11b, CD11c, and CD19 in PBLs and spleens. BM chimeras were re-irradiated and infused with allogeneic WT B6 BM and purified T cells, then monitored daily for survival.

Flow cytometry. The following fluorochrome-conjugated Abs were purchased from eBioscience, BD Biosciences, BioLegend, Abcam, or R\&D Systems: CD4 (RM4-5), CD8 (53-6.7), H-2Kb (AF6-88.5), H-2Kd (SF11.1), CD16/CD32 (clone 93), CD25 (PC61.5), CD62L (MEL-14), CD44 (IM7), CD69 (H1.2F3), LPAM-1 (DATK32), CCR5 (HM-CCR5-7A4), CCR9 (CW-1.2), CXCR3 (CXCR3-173), CD107a (eBio1D4B), B7-H4 (clone 297219), IFN- $\gamma$ (XMG1.2), TNF- $\alpha$ (MP6-XT22), IL-6 (MP5-20F3), IL-22 (IL22JOP), CD45.1 (A20), CD45.2 (clone 104), CD127 (A7R34), CD98 (RL388), CD36 (clone 72-1), CTLA-4 (UC10-4B9), Lag-3 (C9B7W), TIM3 (RMT3-23), CD73 (eBioTY/11.8), 2B4 (eBio244F4), PD-1 (J43), CD71 (R17217), TIGIT (GIGD7), Granzyme B (NGZB), Perforin (eBioOMAK-D), Ki-67 (B56), GLUT1 (ab14683), CPT1A (8F6AE9), and CD178 (MFL3). 7-AAD, Annexin V, and fixable viability dye were purchased from eBioscience. Secondary Abs were purchased from Jackson ImmunoResearch. Carboxyfluorescein diacetate succinimidyl ester (CFSE), Celltrace violet (CTV), Dihydroethidium (DHE), Tetramethylrhodamine (TMRM, methyl ester), MitoTracker Green (MTG), MitoTracker Deep Red (MTDR), and BoDipy (500/510 $\mathrm{C}_{1}$, $\mathrm{C}_{12}$ ) were purchased from Thermo Fisher Scientific. Cells were stained with viability dye to discriminate live from dead cells. Cell apoptosis was measured using Annexin V staining kit. Superoxide production by 
donor T cells was measured by flow cytometry using DHE (a redox-sensitive dye specific for superoxide) as described previously (61). MTG and MTDR staining (62) were performed according to manufacturer's instructions. Intracellular cytokine staining was performed as described previously (30). Isotype-matched Abs were used as control. Cells were acquired using BD LSRFortessa flow cytometer (BD Biosciences) and data were analyzed using FlowJo software. Responder frequency and proliferation capacity of donor $\mathrm{CD} 4^{+}$ and $\mathrm{CD} 8^{+} \mathrm{T}$ cells were calculated as described previously (63).

Treg suppression assay. Tregs were isolated from LNs of WT BALB/c or B7-H4 ${ }^{-1-}$ mice by MACS column selection to more than 93\% purity. Splenocytes isolated from B6 Ly5.2 mice were labeled with CFSE and used as responder cells. Responder cells were stimulated with purified anti-mCD3e $\mathrm{mAb}(0.25 \mu \mathrm{g} / \mathrm{mL}$; eBioscience) and were cultured with or without freshly isolated Tregs at the indicated $\mathrm{T}$ cell/Treg ratios. Cells were harvested on day 4 , and proliferation was determined by CFSE dilution.

Bioluminescence imaging (BLI) studies. Xenogen IVIS imaging system was used for in vivo imaging (30). Mice were injected i.p. with firefly luciferase substrate $(0.1 \mathrm{~mL} ; 30 \mathrm{mg} / \mathrm{mL}$; Perkin Elmer $5 \mathrm{~min}$ utes before imaging. Data were analyzed using Living Image Software (Perkin Elmer) and presented as photon counts per area.

Histology. Representative mice were sacrificed on day 21 or day 30 after BMT and harvested organs were embedded in OCT compound, snap-frozen in liquid nitrogen, and stored at $-80^{\circ} \mathrm{C}$. Lungs were inflated by infusion of $1 \mathrm{~mL}$ of OCT compound: PBS (3:1) intratracheally before harvest. Cryosections $(6-\mu \mathrm{m})$ were fixed in acetone, stained with $\mathrm{H} \& \mathrm{E}$ and histopathology scores were assigned as described previously (64).

FITC-dextran permeability assay. FITC-dextran assay was used to evaluate intestinal permeability as described previously (30). Briefly, mice were given 16 mg of FITC-dextran (Sigma-Aldrich) orally; 4 hours later, plasma was collected, and fluorescence intensity of each sample was measured on a plate reader.

Colon lymphocyte isolation. Mice were sacrificed on day 23 or day 29 after BMT, colons were harvested, cut into pieces, and incubated 3 to 4 times with 5 mM EDTA in RPMI 1640 with $10 \%$ serum at $37^{\circ} \mathrm{C}$ for 15 minutes. Supernatants containing intraepithelial lymphocytes were discarded. Tissues were then incubated twice with $0.5 \mathrm{mg} / \mathrm{mL}$ and once with $1 \mathrm{mg} / \mathrm{mL}$ of Collagenase D (Roche) in RPMI 1640 with $10 \%$ serum at $37^{\circ} \mathrm{C}$ for 1 hour. Lymphocytes were isolated on a 40/80 Percoll (Sigma-Aldrich) gradient (38).

Metabolism assays. Experiments were performed as described previously (38). Briefly, mice were sacrificed on day 4 or day 5 after BMT and donor T cells were purified from spleens (3 spleens were pooled to make 1 pooled sample and 4 pooled samples/group). ECAR and OCR of purified donor T cells were measured with a XF-24 Extracellular Flux Analyzer (Seahorse Bioscience). Cells were seeded in a Cell-Tak coated plate and ECAR was measured in XF media with glutamine alone under basal condition and in response to glucose, oligomycin, and 2-deoxyglucose (2-DG). Also, cells were seeded in a Cell-Tak coated plate and OCR was measured in XF media under basal condition and in response to oligomycin, fluorocarbonyl cyanide phenylhydrazone (FCCP), and rotenone + antimycin A. In selected experiments, OCR was also measured in the presence of Etomoxir (Sigma-Aldrich).

Lymphoma cell lines and GVL studies. A20 is a B cell lymphoma cell line of BALB/c origin that was modified to express LUC (termed A20 ${ }^{\text {luc }}$ ) as described previously (65). Lethally irradiated WT BALB/c recipients were infused with $10^{7} \mathrm{~T}$ cell-depleted WT B6 BM cells plus $1 \times 10^{6} \mathrm{~A} 20^{\text {luc }}$-lymphoma cells, or lethally irradiated WT BALB/c or B7-H4 ${ }^{-/}$recipients were infused with $10^{7} \mathrm{~T}$ cell-depleted WT B6 BM cells plus $1 \times 10^{6} \mathrm{~A} 20^{\text {luc }}$-lymphoma cells along with $0.5 \times 10^{6} \mathrm{WT}$ B6 purified T cells on day 0 . Tumor growth was monitored weekly by LUC imaging of lymphoma cells.

TBL-12 is a B cell lymphoma cell line of B6 origin (66) that was modified to express LUC (termed TBL-12 $2^{l u c}$ ). Lethally irradiated B6 recipients were infused with $10^{7} \mathrm{~T}$ cell-depleted WT BALB/c BM cells plus $5 \times 10^{3} \mathrm{TBL}-12^{\text {luc }}$-lymphoma cells, or lethally irradiated B6 recipients were infused with $10^{7}$ T cell-depleted WT BALB/c BM cells plus $5 \times 10^{3}$ TBL- $12^{\text {luc }}-1$ ymphoma cells along with $2 \times 10^{6}$ or $3 \times$ $10^{6} \mathrm{WT}$ BALB/c or B7-H4 $4^{-1}$ purified T cells on day 0 . Tumor growth was monitored weekly by LUC imaging of lymphoma cells.

Statistics. The Kaplan-Meier product-limit method was used to calculate survival and differences between groups was determined using log-rank test (GraphPad Prism 7.0 software). For all other data, 2-tailed Student's $t$ test was used to analyze differences between groups. $P<0.05$ was considered as significant.

Study approval. All experiments were conducted in accordance with approved protocols by Institutional Animal Care and Use Committee (IACUC) at the University of Minnesota. 


\section{Author contributions}

AS designed and performed experiments, analyzed data, and wrote the manuscript. PAT, CJL, MJO, CJF, GT, WM, and KK performed experiments. APM assigned pathology scoring. YR, CD, SH, and XZ provided key biological materials. GRH, DHM, WJM, JSS, IM, MVDB, JPA, and RZ provided data discussion and edited the manuscript. BRB designed experiments, discussed data, and edited the manuscript.

\section{Acknowledgments}

The authors thank David A. Bernlohr and Rocio Foncea (Department of Biochemistry, Molecular Biology and Biophysics, University of Minnesota) and the Minnesota Obesity Center (NIH P30 DK050456) for assistance in Seahorse experiments, and Jamie Panthera and Josh Dysthe for animal handling. Bioluminescence imaging studies were performed using Xenogen IVIS imaging system and staff support at the University of Minnesota imaging center (http://uic.umn.edu). This study was supported in part by NIH grants HL056067, AIO34495, and AIO56299 (to BRB).

Address correspondence to: Bruce R. Blazar, University of Minnesota, Department of Pediatrics, MMC 109, 420 Delaware Street Southeast, Minneapolis, Minnesota 55455, USA. Phone: 612.626.1926; Email: blaza001@umn.edu.

1. Socié G, Blazar BR. Acute graft-versus-host disease: from the bench to the bedside. Blood. 2009;114(20):4327-4336.

2. Zeiser R, Blazar BR. Acute graft-versus-host disease - biologic process, prevention, and therapy. N Engl J Med. 2017;377(22):2167-2179.

3. Prasad DV, Richards S, Mai XM, Dong C. B7S1, a novel B7 family member that negatively regulates T cell activation. Immunity. 2003;18(6):863-873.

4. Sica GL, et al. B7-H4, a molecule of the B7 family, negatively regulates T cell immunity. Immunity. 2003;18(6):849-861.

5. Zang X, Loke P, Kim J, Murphy K, Waitz R, Allison JP. B7x: a widely expressed B7 family member that inhibits T cell activation. Proc Natl Acad Sci U S A. 2003;100(18):10388-10392.

6. Choi IH, et al. Genomic organization and expression analysis of B7-H4, an immune inhibitory molecule of the B7 family. J Immunol. 2003;171(9):4650-4654.

7. Wei J, Loke P, Zang X, Allison JP. Tissue-specific expression of B7x protects from CD4 T cell-mediated autoimmunity. J Exp Med. 2011;208(8):1683-1694.

8. Tringler B, et al. B7-h4 is highly expressed in ductal and lobular breast cancer. Clin Cancer Res. 2005;11(5):1842-1848.

9. Krambeck AE, et al. B7-H4 expression in renal cell carcinoma and tumor vasculature: associations with cancer progression and survival. Proc Natl Acad Sci U S A. 2006;103(27):10391-10396.

10. Lee JS, et al. B7x in the periphery abrogates pancreas-specific damage mediated by self-reactive CD8 T cells. J Immunol. 2012;189(8):4165-4174.

11. Wang X, et al. Local expression of B7-H4 by recombinant adenovirus transduction in mouse islets prolongs allograft survival. Transplantation. 2009;87(4):482-490.

12. Xu JF, et al. Ectopic B7-H4-Ig expression attenuates concanavalin A-induced hepatic injury. Clin Immunol. 2010;136(1):30-41.

13. Wang X, et al. Early treatment of NOD mice with B7-H4 reduces the incidence of autoimmune diabetes. Diabetes. 2011;60(12):3246-3255.

14. Podojil JR, et al. B7-H4Ig inhibits mouse and human T-cell function and treats EAE via IL-10/Treg-dependent mechanisms. J Autoimmun. 2013;44:71-81.

15. Lee IF, et al. B7-H4.Ig inhibits the development of type 1 diabetes by regulating Th17 cells in NOD mice. Cell Immunol. 2013;282(1):1-8.

16. Radichev IA, et al. Loss of peripheral protection in pancreatic islets by proteolysis-driven impairment of VTCN1 (B7-H4) presentation is associated with the development of autoimmune diabetes. J Immunol. 2016;196(4):1495-1506.

17. Hofmeyer KA, Scandiuzzi L, Ghosh K, Pirofski LA, Zang X. Tissue-expressed B7x affects the immune response to and outcome of lethal pulmonary infection. J Immunol. 2012;189(6):3054-3063.

18. Zhu G, et al. B7-H4-deficient mice display augmented neutrophil-mediated innate immunity. Blood. 2009;113(8):1759-1767.

19. Podojil JR, Miller SD. Potential targeting of B7-H4 for the treatment of cancer. Immunol Rev. 2017;276(1):40-51.

20. Radichev IA, et al. Nardilysin-dependent proteolysis of cell-associated VTCN1 (B7-H4) marks type 1 diabetes development. Diabetes. 2014;63(10):3470-3482.

21. Kryczek I, et al. B7-H4 expression identifies a novel suppressive macrophage population in human ovarian carcinoma. $J$ Exp Med. 2006;203(4):871-881.

22. Taylor PA, Lees CJ, Blazar BR. The infusion of ex vivo activated and expanded CD4(+)CD25(+) immune regulatory cells inhibits graft-versus-host disease lethality. Blood. 2002;99(10):3493-3499.

23. Petrovic A, et al. LPAM ( $\alpha 4 \beta 7$ integrin) is an important homing integrin on alloreactive T cells in the development of intestinal graft-versus-host disease. Blood. 2004;103(4):1542-1547.

24. Wysocki CA, Panoskaltsis-Mortari A, Blazar BR, Serody JS. Leukocyte migration and graft-versus-host disease. Blood. 2005;105(11):4191-4199.

25. Brandl K, et al. Enhanced sensitivity to DSS colitis caused by a hypomorphic Mbtps1 mutation disrupting the ATF6-driven unfolded protein response. Proc Natl Acad Sci U S A. 2009;106(9):3300-3305. 
26. Nguyen HD, Kuril S, Bastian D, Yu XZ. T cell metabolism in hematopoietic cell transplantation. Front Immunol. 2018;9:176.

27. MacIver NJ, Michalek RD, Rathmell JC. Metabolic regulation of T lymphocytes. Annu Rev Immunol. 2013;31:259-283.

28. Maciver NJ, Jacobs SR, Wieman HL, Wofford JA, Coloff JL, Rathmell JC. Glucose metabolism in lymphocytes is a regulated process with significant effects on immune cell function and survival. J Leukoc Biol. 2008;84(4):949-957.

29. Gatza E, et al. Manipulating the bioenergetics of alloreactive T cells causes their selective apoptosis and arrests graft-versus-host disease. Sci Transl Med. 2011;3(67):67ra8.

30. Saha A, et al. Host programmed death ligand 1 is dominant over programmed death ligand 2 expression in regulating graft-versus-host disease lethality. Blood. 2013;122(17):3062-3073.

31. Byersdorfer CA, et al. Effector T cells require fatty acid metabolism during murine graft-versus-host disease. Blood. 2013;122(18):3230-3237.

32. Silverstein RL, Febbraio M. CD36, a scavenger receptor involved in immunity, metabolism, angiogenesis, and behavior. Sci Signal. 2009;2(72):re3.

33. Li J, et al. Co-inhibitory molecule B7 superfamily member 1 expressed by tumor-infiltrating myeloid cells induces dysfunction of anti-tumor $\mathrm{CD}^{+} \mathrm{T}$ cells. Immunity. 2018;48(4):773-786.e5

34. Taylor PA, Noelle RJ, Blazar BR. CD4(+)CD25(+) immune regulatory cells are required for induction of tolerance to alloantigen via costimulatory blockade. J Exp Med. 2001;193(11):1311-1318.

35. Quandt D, Fiedler E, Boettcher D, Marsch WCh, Seliger B. B7-h4 expression in human melanoma: its association with patients' survival and antitumor immune response. Clin Cancer Res. 2011;17(10):3100-3111.

36. Qian Y, Shen L, Cheng L, Wu Z, Yao H. B7-H4 expression in various tumors determined using a novel developed monoclonal antibody. Clin Exp Med. 2011;11(3):163-170.

37. Chen C, et al. Induced expression of B7-H4 on the surface of lung cancer cell by the tumor-associated macrophages: a potential mechanism of immune escape. Cancer Lett. 2012;317(1):99-105

38. Saha A, et al. Programmed death ligand-1 expression on donor T cells drives graft-versus-host disease lethality. J Clin Invest. 2016;126(7):2642-2660.

39. Veenstra RG, et al. B7-H3 expression in donor T cells and host cells negatively regulates acute graft-versus-host disease lethality. Blood. 2015;125(21):3335-3346.

40. Wu SR, Reddy P. Tissue tolerance: a distinct concept to control acute GVHD severity. Blood. 2017;129(13):1747-1752.

41. Blazar BR, et al. Blockade of programmed death-1 engagement accelerates graft-versus-host disease lethality by an IFN-gamma-dependent mechanism. J Immunol. 2003;171(3):1272-1277.

42. Taylor PA, Lees CJ, Fournier S, Allison JP, Sharpe AH, Blazar BR. B7 expression on T cells down-regulates immune responses through CTLA-4 ligation via T-T interactions [corrections]. J Immunol. 2004;172(1):34-39.

43. Jeon H, et al. Structure and cancer immunotherapy of the B7 family member B7x. Cell Rep. 2014;9(3):1089-1098

44. Chetoui N, Boisvert M, Gendron S, Aoudjit F. Interleukin-7 promotes the survival of human CD4 ${ }^{+}$effector/memory T cells by up-regulating Bcl-2 proteins and activating the JAK/STAT signalling pathway. Immunology. 2010;130(3):418-426.

45. Kaech SM, Tan JT, Wherry EJ, Konieczny BT, Surh CD, Ahmed R. Selective expression of the interleukin 7 receptor identifies effector CD8 T cells that give rise to long-lived memory cells. Nat Immunol. 2003;4(12):1191-1198.

46. Li J, Huston G, Swain SL. IL-7 promotes the transition of CD4 effectors to persistent memory cells. J Exp Med. 2003;198(12):1807-1815.

47. Snider D, Liang H. Early intestinal Th1 inflammation and mucosal T cell recruitment during acute graft-versus-host reaction. J Immunol. 2001;166(10):5991-5999.

48. Reddy $\mathrm{P}$, et al. Interleukin-18 regulates acute graft-versus-host disease by enhancing Fas-mediated donor T cell apoptosis. $J$ Exp Med. 2001;194(10):1433-1440.

49. Burman AC, et al. IFNgamma differentially controls the development of idiopathic pneumonia syndrome and GVHD of the gastrointestinal tract. Blood. 2007;110(3):1064-1072.

50. Caro-Maldonado A, Gerriets VA, Rathmell JC. Matched and mismatched metabolic fuels in lymphocyte function. Semin Immunol. 2012;24(6):405-413.

51. Wang R, Green DR. Metabolic checkpoints in activated T cells. Nat Immunol. 2012;13(10):907-915.

52. Chang $\mathrm{CH}$, et al. Posttranscriptional control of T cell effector function by aerobic glycolysis. Cell. 2013;153(6):1239-1251.

53. Kidani Y, et al. Sterol regulatory element-binding proteins are essential for the metabolic programming of effector $\mathrm{T}$ cells and adaptive immunity. Nat Immunol. 2013;14(5):489-499.

54. Sinclair LV, Rolf J, Emslie E, Shi YB, Taylor PM, Cantrell DA. Control of amino-acid transport by antigen receptors coordinates the metabolic reprogramming essential for T cell differentiation. Nat Immunol. 2013;14(5):500-508.

55. Michalek RD, et al. Cutting edge: distinct glycolytic and lipid oxidative metabolic programs are essential for effector and regulatory CD4+ T cell subsets. J Immunol. 2011;186(6):3299-3303.

56. Macintyre AN, et al. The glucose transporter Glut1 is selectively essential for CD4 T cell activation and effector function. Cell Metab. 2014;20(1):61-72.

57. Zhang DX, Gutterman DD. Mitochondrial reactive oxygen species-mediated signaling in endothelial cells. Am J Physiol Heart Circ Physiol. 2007;292(5):H2023-H2031.

58. Lambert AJ, Brand MD. Reactive oxygen species production by mitochondria. Methods Mol Biol. 2009;554:165-181.

59. O'Sullivan D, et al. Memory CD8(+) T cells use cell-intrinsic lipolysis to support the metabolic programming necessary for development. Immunity. 2014;41(1):75-88.

60. Cooke KR, et al. An experimental model of idiopathic pneumonia syndrome after bone marrow transplantation: I. The roles of minor H antigens and endotoxin. Blood. 1996;88(8):3230-3239.

61. Blatt NB, et al. Benzodiazepine-induced superoxide signals B cell apoptosis: mechanistic insight and potential therapeutic utility. J Clin Invest. 2002;110(8):1123-1132.

62. Zhou R, Yazdi AS, Menu P, Tschopp J. A role for mitochondria in NLRP3 inflammasome activation. Nature. 2011;469(7329):221-225.

63. Wells AD, Gudmundsdottir H, Turka LA. Following the fate of individual T cells throughout activation and clonal expansion. 
Signals from $\mathrm{T}$ cell receptor and CD28 differentially regulate the induction and duration of a proliferative response. J Clin Invest. 1997;100(12):3173-3183.

64. Blazar BR, et al. Engraftment of severe combined immune deficient mice receiving allogeneic bone marrow via In utero or postnatal transfer. Blood. 1998;92(10):3949-3959.

65. Taylor PA, et al. Insights into the mechanism of FTY720 and compatibility with regulatory T cells for the inhibition of graftversus-host disease (GVHD). Blood. 2007;110(9):3480-3488.

66. Bjordahl RL, Gapin L, Marrack P, Refaeli Y. iNKT cells suppress the CD8+ T cell response to a murine Burkitt's-like B cell lymphoma. PLoS One. 2012;7(8):e42635. 